\title{
Proof of a conjecture of Galvin
}

\author{
Dilip Raghavan ${ }^{1}$ and Stevo Todorcevic ${ }^{2,3}$ \\ ${ }^{1}$ Department of Mathematics, National University of Singapore, Singapore 119076; E-mail: dilip.raghavan@protonmail.com. \\ ${ }^{2}$ Department of Mathematics, University of Toronto, Toronto, ON, Canada, M5S 2E4; E-mail: stevo@ math.toronto.edu. \\ ${ }^{3}$ Institut de Mathématique de Jussieu, UMR 7586, Case 247, 4 place Jussieu, 75252 Paris Cedex, France; \\ E-mail: todorcevic@math.jussieu.fr.
}

Received: 14 January 2020; Revised: 17 November 2020; Accepted: 16 October 2020

MSC Codes: Primary - 03E02; Secondary - 05D10; 03E55, 05C55, 54E40 (others)

\begin{abstract}
We prove that if the set of unordered pairs of real numbers is coloured by finitely many colours, there is a set of reals homeomorphic to the rationals whose pairs have at most two colours. Our proof uses large cardinals and verifies a conjecture of Galvin from the 1970s. We extend this result to an essentially optimal class of topological spaces in place of the reals.
\end{abstract}

\section{Contents}

1 Introduction

2 Ramsey-degree calculus 2

3 Ramsey degrees within a wider class of spaces $\quad \mathbf{5}$

4 Notation $\quad \mathbf{8}$

5 Some preliminaries $r$

6 Main theorem 11

\section{Introduction}

In this paper we present a result that sheds light on a general problem about the behavior of an arbitrary relational structure of the form $\left(\mathbb{R}, S_{1}, \ldots, S_{n}\right)$ on 'large' subsets of $\mathbb{R}$. A general result of Ehrenfeucht and Mostowski [4], anticipated already in the seminal paper of Ramsey [20], shows that such problems can be reduced to problems about finite colourings of the symmetric cubes $[\mathbb{R}]^{k}$ (the set of all $k$-element sets of real numbers), where the integer $k$ is closely related to the arity of the (finite list of) given relations on $\mathbb{R}$. In other words, in our general problem we could restrict ourselves to relational structures of the form $(\mathbb{R}, E)$, where $E$ is a single equivalence relation with finitely many equivalence classes on an appropriate symmetric cube $[\mathbb{R}]^{k}$. Answering a question of Knaster, Sierpiński [23] showed in 1933 that a well-ordering $<_{\text {wo }}$ of $\mathbb{R}$ can be used in defining a particular equivalence relation $E_{k}^{S}$ on $[\mathbb{R}]^{k}$ with $k !(k-1)$ ! classes by comparing the behaviors of the well-ordering $<_{\text {wo }}$ and the usual ordering on a given $k$-element set $s$ as well as recording the ordering of distances between consecutive elements of $s$ when enumerated increasingly according to the usual ordering of $\mathbb{R}$. What Sierpiński's proof shows is that the number $k !(k-1)$ ! of equivalence classes of $E_{k}^{S}$ cannot be reduced by restricting it to any uncountable or, more generally, nonempty and dense-in-itself subset of $\mathbb{R}$. This feature of Sierpiński's proof was first

(C) The Author(s), 2020. Published by Cambridge University Press. This is an Open Access article, distributed under the terms of the Creative Commons Attribution licence (http://creativecommons.org/licenses/by/4.0/), which permits unrestricted re-use, distribution, and reproduction in any medium, provided the original work is properly cited. 
put forward by Galvin in a letter to Laver [9], and it was reiterated some years later when Baumgartner proved that in this problem $\mathbb{R}$ cannot be replaced by any countable topological space. Baumgartner [1, page 182] explicitly states the 2-dimensional version of Galvin's conjecture solved here, along with an opinion that this is probably the most interesting open problem in this area. We will show using large cardinals that if $X$ is an arbitrary uncountable set of reals and $E$ is an equivalence relation on $[X]^{2}$, then there is $Y \subseteq X$ homeomorphic to $\mathbb{Q}$ such that $E \uparrow[Y]^{2}$ is coarser than $E_{2}^{S} \uparrow[Y]^{2}$. In fact, we shall isolate what appears to be the optimal general topological condition on the space $X$ that guarantees this conclusion, with $E_{2}^{S}$ replaced by an appropriate analogous equivalence relation on $[X]^{2}$.

We finish this introduction with comments on the methods behind the proofs of these results. Given a space $X$ satisfying certain conditions and a finite colouring $c:[X]^{2} \rightarrow l$, we use large cardinals to construct a topological copy $Y \subseteq X$ of $\mathbb{Q}$ such that $[Y]^{2}$ uses no more than two colours. In hindsight, the conditions on $X$ are made in order to allow us a construction using large cardinals of another space $Z$ together with a continuous map $f: Z \rightarrow X$ such that $Z$ is a Baire space, $f$ is not constant on any nonempty open subset of $Z$ and the induced colouring $c_{f}:[Z]^{2} \rightarrow l+1-$ given by $c_{f}(x, y)=c(f(x), f(y))$ if $f(x) \neq f(y)$ and $c_{f}(x, y)=l$ if $f(x)=f(y)-$ is in some weak sense Baire measurable. Thus the problem is transferred to $Z$, where it becomes possible to use Banach-Mazur games to construct a copy of $\mathbb{Q}$ which uses only two colours of $c_{f}$ and on which $f$ is one-to-one. The conditions on $X$ which allow us (using large cardinals) such transfer to a Baire space $Z$ and a continuous nowhere constant map $f$ were already used in [28], which in turn was motivated by a problem of Haydon [11] from the theory of differentiability in the context of general Banach spaces. It should also be noted that large cardinals are introduced into the construction of $Z$ and $f: Z \rightarrow X$ through the ideas behind the stationary tower forcing of Woodin [32], which in turn was inspired by the groundbreaking work of Foreman, Magidor and Shelah [8]. The only use of the hypothesis on the existence of large cardinals is Theorem 28, which is equivalent to the well-known fact that the stationary tower up to a suitably large cardinal is precipitous. It is also worth noting that Theorem 28 is applied only once in the proof of the main result, to get Lemma 44.

We believe that applying large cardinals to structural Ramsey theory is a new idea that will give us more results of this kind. We do not know if large cardinals are necessary for the results in this paper. However, it would not be surprising if some large cardinal is needed for our main Theorem 17, in view of the fact that large cardinals are provably required for a result from [28] which establishes the nonexistence of a certain kind of topological space of size $\boldsymbol{\aleph}_{1}$ through an application of Theorem 28.

Finally, we mention that the precise forms of our results are explained in Sections 2 and 3, where we comment on their general interest and how they are related to other areas of mathematics.

\section{Ramsey-degree calculus}

In this section we state the general form of our result for sets of reals, putting it into the context of other results in this area. Suppose that $\mathcal{C}$ is some class of structures and $A$ is a structure that embeds into every member of $\mathcal{C}$. One of the goals of Ramsey theory is to find, for each natural number $k \geq 1$, the smallest number $t_{k}$ such that for every natural number $l \geq 1$, every structure $B \in \mathcal{C}$ and every colouring that assigns one of $l$ colours to each $k$-element subset of $B$, there exists a substructure $X \subseteq B$ which is isomorphic to $A$ and has the property that at most $t_{k}$ colours occur among the $k$-element subsets of $X$. This natural number $t_{k}$, if it exists, is called the $k$-dimensional Ramsey degree of the structure $A$ within the class $\mathrm{C}$.

The problem of computing the Ramsey degrees of $A$ in a class of structures $\mathcal{C}$ can be formulated as an expansion problem. Let us say that $R$ is a finitary relation on $A$ to mean that there is an integer $k \geq 1$ so that $R$ consists of $k$-tuples from $A$. The expansion problem for $A$ within $\mathcal{C}$ asks for a list of finitely many finitary relations $R_{1}, \ldots, R_{n}$ on $A$ that are atomic for the structures in $\mathcal{C}$ in the following sense: to each structure $B \in \mathcal{C}$ and an arbitrary finitary relation $S$ on $B$, it is possible to associate a substructure $X \subseteq B$ and an isomorphism $\varphi: A \rightarrow X$ such that the restriction of $S$ to $X$ is definable without quantifiers from the images of $R_{1}, \ldots, R_{n}$ under $\varphi$. Determining the Ramsey degrees of $A$ within $\mathcal{C}$ solves the expansion 
problem for $A$ within $\mathcal{C}$. Frequently, the atomic finitary relations that solve an expansion problem turn out to be purely order-theoretic in nature. An example of such a computation of canonical forms for arbitrary finitary relations on $\mathbb{N}$ via Ramsey's original theorem can be found in [29, Theorem 1.7]. This computation was originally done by Ramsey in [20]; a closely related result was rediscovered by Erdôs and Rado [5]. It should be clear that the richer the structure of $A$ is, the more informative is a solution to the expansion problem for $A$ in $\mathcal{C}$.

Expansion problems for various pairs $\langle A, \mathcal{C}\rangle$ occur frequently in topological dynamics in the form of questions about representations of the universal minimal flow of the automorphism group of an ultrahomogeneous structure. See [15] for further details on the connections between Ramsey theory and topological dynamics of automorphism groups, where a precise correspondence is given between Ramsey-degree calculus and representation theory for universal minimal flows of such groups.

In this paper, it will be proved, assuming large cardinals, that the 2-dimensional Ramsey degree of the topological space $\mathbb{Q}$ of the rationals within the class of all regular, non-left-separated spaces with a point-countable base is at most 2 . Our result is provably optimal for metrisable spaces. The following terminology will make certain results easier to state:

Definition 1. Let $X$ be any set. For any cardinal number $\kappa,[X]^{\kappa}$ is the collection of subsets of $X$ of cardinality $\kappa$, and $[X]^{<\kappa}$ denotes the collection of subsets of $X$ that have cardinality less than $\kappa$.

Let $X$ and $Y$ be topological spaces. For natural numbers $k, l, t \geq 1$, we will write

$$
X \rightarrow(Y)_{l, t}^{k}
$$

to mean that for every set $L$ of cardinality $l$ and every colouring $c:[X]^{k} \rightarrow L$, there exist a subspace $Y^{\prime} \subseteq X$ homeomorphic to $Y$ and a subset $T \subseteq L$ of cardinality $t$ such that $\left\{c(v): v \in\left[Y^{\prime}\right]^{k}\right\} \subseteq T$. If $t=1$, then it is not recorded in this notation - that is, we write $X \rightarrow(Y)_{l}^{k}$ instead of $X \rightarrow(Y)_{l, 1}^{k}$.

For a natural number $k \geq 1$, the $k$-dimensional Ramsey degree of a space $Y$ inside the space $X$, if it exists, is the least natural number $t \geq 1$ with the property that $X \rightarrow(Y)_{l, t}^{k}$ for all $l<\omega$.

When $X$ is a linearly ordered topological space, this notation is ambiguous, but that will cause no confusion in what follows.

We introduce the following abbreviations in order to succinctly state the large-cardinal hypothesis in our main results:

Definition 2. ZFC stands for Zermelo Frankel set theory with the axiom of Choice. For an ordinal $\alpha$, let $\left(*_{\alpha}\right)$ denote the following statement:

There is an inner model $N$ of ZFC such that $V_{\alpha} \subseteq N$, and in $N$ there is a Woodin cardinal greater than $\alpha$.

Let $(*)$ be the statement that for every ordinal $\alpha,\left(*_{\alpha}\right)$ holds.

Using the terminology of Definitions 1 and 2, one of the important consequences of the main result of this paper may be stated as follows:

Theorem 3. Assume (*). Let $X$ be a non- $\sigma$-discrete metric space. Then the 2-dimensional Ramsey degree of $\mathbb{Q}$ in $X$ is 2 .

Corollary 4. Assume that there is either a proper class of Woodin cardinals or a strongly compact cardinal. Let $X$ be a non- $\sigma$-discrete metric space. Then the 2-dimensional Ramsey degree of $\mathbb{Q}$ in $X$ is 2.

That non- $\sigma$-discreteness is an optimal restriction in this theorem and its corollary follows from the next result: 
Theorem 5 [30]. If $X$ is a $\sigma$-discrete metric space, then there is $c:[X]^{2} \rightarrow \omega$ such that $c^{\prime \prime}[Y]^{2}=\omega$ for all $Y \subseteq X$ homeomorphic to $\mathbb{Q}$.

It follows that the Ramsey degree of $\mathbb{Q}$ does not exist (is infinite) in any $\sigma$-discrete metric space. The equivalence stated in the following corollary encapsulates Corollary 4 and the fact that it is optimal for metrisable spaces:

Corollary 6. Assume that there is either a proper class of Woodin cardinals or a strongly compact cardinal. Then the following are equivalent for every metrisable space $X$ :

(a) $X$ is not $\sigma$-discrete.

(b) $X \rightarrow(\mathbb{Q})_{l, 2}^{2}$ for every natural number $l \geq 1$.

Note that condition (a) is equivalent to $X \rightarrow(\omega+1)_{\omega}^{1}$, so we have here an analogy between Corollary 6 and a theorem of the second author from [24], stating that for any partial order $P, P \rightarrow(\omega)_{\omega}^{1}$ if and only if $P \rightarrow(\alpha)_{k}^{2}$ for all $\alpha<\omega_{1}$ and $k<\omega$.

We will briefly comment on the large-cardinal hypotheses of Theorem 3 and Corollary 4 . It is possible that the universe contains a strongly compact cardinal which also happens to be the only measurable cardinal (see Magidor [18]). Therefore, the two disjuncts in the hypothesis of Corollary 4 are formally independent of each other, even though the consistency strength of a strongly compact cardinal is much greater than that of a proper class of Woodin cardinals. Nevertheless, the hypothesis $(*)$, though more technical in nature, is weaker than either of these disjuncts. The hypothesis $(*)$ is implied by each of the following: the existence of a proper class of Woodin cardinals, the existence of one strongly compact cardinal and the Proper Forcing Axiom (PFA). The hypothesis (*) can be immediately obtained from the existence of a strongly compact cardinal using the results of Jensen and Steel [13]. In fact, it is a theorem (see [31, Theorem .04]) that if one assumes that $\square_{\lambda^{+}}$fails for a proper class of singular strong limit cardinals $\lambda$, then for every ordinal $\alpha$ there is an inner model $N$ of ZFC such that $V_{\alpha} \subseteq N$ and in $N$ there is a proper class of Woodin cardinals. Furthermore, $(*)$ is an entirely local statement that does not even imply the existence of an inaccessible cardinal in $V$. Thus it is much weaker than either the existence of a strongly compact cardinal or the existence of a proper class of Woodin cardinals, although not in consistency strength. Interestingly, $(*)$ turns out to be equivalent to the statement that $\Sigma_{2}^{1}$-determinacy holds in $V$ and all of its set generic extensions (see [33]).

The special case of Theorem 3 restricted to uncountable sets $X \subseteq \mathbb{R}$ is particularly interesting because in this case it is simple to describe a colouring that uses both colours on every dense-in-itself subset of $X$. Recall how Sierpiński's colouring $s:[\mathbb{R}]^{2} \rightarrow\{0,1\}$ is defined by comparing a well-ordering of the reals $<_{\text {wo }}$ with the usual ordering <: given a pair $\{x, y\} \in[\mathbb{R}]^{2}$, we assign $s(\{x, y\})=0$ if and only if $<_{\text {wo }}$ and $<$ agree on $\{x, y\}$. To see that this colouring establishes $\mathbb{R} \rightarrow(\mathbb{Q})_{2}^{2}$, note that any monochromatic subset of $\mathbb{R}$ must be either well-ordered or reverse well-ordered by $<$. Hence no subset of $\mathbb{R}$ which contains a $\mathbb{Z}$-chain in the usual ordering can be monochromatic. Let $E^{S}$ be the equivalence relation on $[\mathbb{R}]^{2}$ that has the two sets $s^{-1}(i)(i<2)$ as equivalence classes. The restriction of Corollary 4 to uncountable sets $X \subseteq \mathbb{R}$ implies that every equivalence relation on $[X]^{2}$ with finitely many classes must be coarser than $E^{S}$ on a copy of $\mathbb{Q}$. A single Woodin cardinal suffices for this restriction.

Corollary 7. Assume that there is either a Woodin cardinal or a strongly compact cardinal. Let $X$ be an uncountable set of reals. Then for every equivalence relation $E$ on $[X]^{2}$ with finitely many equivalence classes, there is $Y \subseteq X$ homeomorphic to $\mathbb{Q}$ such that $E \uparrow[Y]^{2}$ is coarser than $E^{S} \uparrow[Y]^{2}$.

Proof. To see this, let $l \geq 1$ be a natural number and let $c:[X]^{2} \rightarrow l$ be a colouring which gives us an equivalence relation on $[X]^{2}$ with $l$ classes. Define a new colouring $d:[X]^{2} \rightarrow l \times 2$ by setting $d(\{x, y\})=\langle c(\{x, y\}), s(\{x, y\})\rangle$, for every $\{x, y\} \in[X]^{2}$. Here $s$ is Sierpiński's colouring already defined from an arbitrary well-ordering of $\mathbb{R}$. Applying Corollary 4, there must be a set $Y \subseteq X$ as well 
as colours $i, j<l$ such that $Y$ is homeomorphic to $\mathbb{Q}$ and $\left\{c(v): v \in[Y]^{2}\right\} \subseteq\{\langle i, 0\rangle,\langle j, 1\rangle\}$. If $i=j$, then $c$ is constant on $[Y]^{2}$. And if $i \neq j$, then $c$ is equivalent to $s$ on $[Y]^{2}$, with the colour $i$ playing the role of the colour 0 of $s$ and $j$ playing the role of 1 .

Theorem 3 also implies that any well-ordering < $<_{\text {wo }}$ solves the 2-dimensional expansion problem for $\mathbb{Q}$ within the class of all uncountable sets of reals.

Corollary 8. Assume that there is either a Woodin cardinal or a strongly compact cardinal. Let $<_{\text {wo }}$ be any well-ordering of $\mathbb{R}$ and let $<$ be the usual ordering of $\mathbb{R}$. Then for every uncountable $X \subseteq \mathbb{R}$ and every binary relation $M \subseteq X^{2}$, there exists a set $Y \subseteq X$, which is homeomorphic to $\mathbb{Q}$, such that $M \cap Y^{2}$ is equal to one of the following relations restricted to $Y: \mathrm{T}, \perp,=, \neq,<,>, \leq, \geq,<_{\mathrm{wo}},>_{\mathrm{wo}}, \leq_{\mathrm{wo}}, \geq_{\mathrm{wo}}$, $<\cap<_{\text {wo }},<\cap>_{\text {wo }},>\cap<_{\text {wo }},>\cap>_{\text {wo }}, \leq \cap \leq_{\text {wo }}, \leq \cap \geq_{\text {wo }}, \geq \cap \leq_{\text {wo }}, \geq \cap \geq_{\text {wo }}$.

Proof. This is similar to the proof of [29, Theorem 1.7], except that in the crucial step we use Corollary 4 in place of Ramsey's theorem.

Just as with Corollary 4, Corollaries 7 and 8 follow from the weaker hypothesis that there is an inner model $N$ of ZFC that contains all sets of reals and has a Woodin cardinal relative to it.

A weak form of the conclusion of Theorem 3, when we restrict the class of all non- $\sigma$-discrete metric spaces to the singleton $\{\mathbb{R}\}$, was first conjectured by Galvin in the 1970s [9], and Galvin's conjecture, even in this weak form, remained unproved until our work. In an earlier unpublished note, Galvin had proved that for every colouring of $[\mathbb{Q}]^{2}$ into finitely many colours, there exists a $Y \subseteq \mathbb{Q}$ which is orderisomorphic to $\mathbb{Q}$ such that at most 2 colours occur in $[Y]^{2}$. This was generalised by Laver [3], who showed that for each natural number $k \geq 1$, there exists a number $t_{k}$ with the property that for every colouring of $[\mathbb{Q}]^{k}$ into finitely many colours, there exists a $Y \subseteq \mathbb{Q}$ which is order-isomorphic to $\mathbb{Q}$ such that at most $t_{k}$ colours occur in $[Y]^{k}$. The optimal value of $t_{k}$ was computed by D. C. Devlin [3]. A recent exposition of Devlin's work can be found in [29, Chapter 6].

Baumgartner [1] was the first to prove that a significant difference emerges when the topological structure of $\mathbb{Q}$ is considered instead of its order structure. Note that if a set of reals is homeomorphic to $\mathbb{Q}$, then it contains a subset which is order-isomorphic to $\mathbb{Q}$, but the reverse is false. In [1], Baumgartner established the special case of Theorem 5 saying that $\mathbb{Q}$ fails to have finite Ramsey degree in dimension 2 within any countable metrisable space.

Results of Shelah [21, 22] hinted at the truth of Theorem 4 for the space $\mathbb{R}$. Assuming suitable large cardinals, Shelah constructed a model of set theory where for any natural number $l \geq 1$ and any colouring $c:[\mathbb{R}]^{2} \rightarrow l$, there is an uncountable set $X \subseteq \mathbb{R}$ such that $c$ uses at most two colours on $[X]^{2}$. His result is a consistency result instead of a direct implication from large cardinals. In his model, the cardinality of $\mathbb{R}$ is quite large - for example, it is a fixed point of the $\boldsymbol{\aleph}$-operation - and the colourings of the pairs for sets of reals of size $\aleph_{1}$ are not controlled. Indeed, by a well-known theorem of Todorcevic [25], if $X$ is any set of size $\boldsymbol{\aleph}_{1}$, then there is a colouring of $[X]^{2}$ into $\boldsymbol{\aleph}_{1}$ many colours so that every uncountable subset of $X$ contains a pair of every colour. The Ramsey degrees of $\mathbb{Q}$ in more general topological spaces have not been determined in Shelah's model.

\section{Ramsey degrees within a wider class of spaces}

We begin by observing that there is a natural generalisation of Sierpiński's colouring to any Tychonoff space.

Definition 9. A topological space $\langle X, \mathcal{T}\rangle$ is called a Tychonoff space if it is a $T_{1}$-space and for every $x \in X$ and every closed set $F \subseteq X$ such that $x \notin F$, there exists a continuous function $f: X \rightarrow[0,1]$ such that $f(x)=0$ and $f(y)=1$ for $y \in F$.

It is a well-known fact that every Tychonoff space of weight $\kappa \geq \boldsymbol{\aleph}_{0}$ is embeddable into $[0,1]^{\kappa}$. 
Definition 10. Let $\kappa \geq \boldsymbol{\aleph}_{0}$ be a cardinal. Define an ordering $<_{\operatorname{lex}}$ on $[0,1]^{\kappa}$ by stipulating that for any $f, g \in[0,1]^{\kappa}$,

$$
f<_{\operatorname{lex}} g \leftrightarrow[f \neq g \quad \text { and } \quad f(\Delta(f, g))<g(\Delta(f, g))]
$$

where $<$ denotes the usual order on $[0,1]$ and $\Delta(f, g)=\min \{\alpha<\kappa: f(\alpha) \neq g(\alpha)\}$.

It is easy to see that $<_{\operatorname{lex}}$ is a linear order on $[0,1]^{\kappa}$. Now fix any well-ordering $<_{\text {wo }}$ of $[0,1]^{\kappa}$ and define Sierpiński's colouring $s:\left[[0,1]^{\kappa}\right]^{2} \rightarrow 2$ with respect to $<_{\text {wo }}$ by stipulating that for any $\{f, g\} \in\left[[0,1]^{\kappa}\right]^{2}, s(\{f, g\})=0$ if and only if $<_{\text {wo }}$ and $<_{\text {lex }}$ agree on $\{f, g\}$.

Lemma 11. Suppose $Y \subseteq[0,1]^{\kappa}$ is nonempty and dense in itself. Then there exist $Y_{0}, Y_{1} \subseteq Y$ such that $Y_{0}$ and $Y_{1}$ are both nonempty and dense in themselves, and $\forall y_{0} \in Y_{0} \forall y_{1} \in Y_{1}\left[y_{0}<\operatorname{lex} y_{1}\right]$.

Proof. $Y$, being nonempty and dense in itself, contains at least two distinct elements. Thus $\Delta(Y)=$ $\{\Delta(f, g): f, g \in Y$ and $f \neq g\}$ is a nonempty subset of $\kappa$. Define $\alpha=\min (\Delta(Y))$. Fix $f, g \in Y$ with $f \neq g$ and $\alpha=\Delta(f, g)$. Without loss of generality, $f(\alpha)<g(\alpha)$. Find a rational number $q$ with $f(\alpha)<q<g(\alpha)$, and define $U=\left\{h \in[0,1]^{\kappa}: h(\alpha)<q\right\}$ and $V=\left\{h \in[0,1]^{\kappa}: h(\alpha)>q\right\}$. Both $U$ and $V$ are open subsets of $[0,1]^{\kappa}$. Furthermore, $f \in Y \cap U=Y_{0}$ and $g \in Y \cap V=Y_{1}$. Therefore, $Y_{0}, Y_{1} \subseteq Y$ are both nonempty and dense in themselves. Now consider any $h \in Y_{0}$ and $h^{\prime} \in Y_{1}$. Then $h, h^{\prime} \in Y$ and $h(\alpha)<q<h^{\prime}(\alpha)$. In particular, $h \neq h^{\prime}$ and $\Delta\left(h, h^{\prime}\right) \leq \alpha$. On the other hand, since $h, h^{\prime} \in Y$, $\alpha \leq \Delta\left(h, h^{\prime}\right)$. So $\alpha=\Delta\left(h, h^{\prime}\right)$, and $h\left(\Delta\left(h, h^{\prime}\right)\right)=h(\alpha)<h^{\prime}(\alpha)=h^{\prime}\left(\Delta\left(h, h^{\prime}\right)\right)$, whence $h<_{\text {lex }} h^{\prime}$.

Theorem 12. Suppose $\langle X, \mathcal{T}\rangle$ is a Tychonoff space. The 2-dimensional Ramsey degree of $\mathbb{Q}$ in $X$ is at least 2.

Proof. We may assume that $\langle X, \mathcal{T}\rangle$ is a subspace of $[0,1]^{\kappa}$, for some $\kappa \geq \boldsymbol{\aleph}_{0}$. Let $s:\left[[0,1]^{\kappa}\right]^{2} \rightarrow 2$ be Sierpiński's colouring defined with respect to an arbitrary well-ordering $<_{\text {wo }}$ of $[0,1]^{\kappa}$. Note that any subset of $X$ that is monochromatic with respect to $s$ must be either well-ordered or reverse well-ordered by $<_{\text {lex }}$. However, if $Y \subseteq X$ is nonempty and dense in itself, then by repeated application of Lemma 11 it is possible to construct a set $Z \subseteq Y$ which is a $\mathbb{Z}$-chain with respect to $<_{\text {lex. }}$.

Baumgartner [1] showed that $X \rightarrow(\omega+1)_{2}^{2}$ for every countable topological space $X$, and that on the other hand, $\mathbb{Q} \rightarrow(\omega+1)_{l, 2}^{2}$ for all $l<\omega$. Here $\omega+1$ denotes the ordinal $\omega \cup\{\omega\}$ with its topology induced by the $\in$-ordering on ordinals, and it naturally represents the converging sequence. In other words, Baumgartner's result says that the converging sequence has 2-dimensional Ramsey degree 2 in the class of all countable dense-in-themselves metrisable spaces. It is not difficult to show that if $X$ is any uncountable set of reals, then $X \rightarrow(\omega+1)_{l}^{2}$ for all $l<\omega$. This was generalised in an unpublished note of the second author from 1996 (extending a previous result from [30]) as follows:

Definition 13. Let $\langle X, \mathcal{T}\rangle$ be a topological space. A base $\mathcal{B} \subseteq \mathcal{T}$ is said to be point-countable if for each $x \in X,\{U \in \mathcal{B}: x \in U\}$ is countable.

Theorem 14 [26]. The following are equivalent for an arbitrary regular space $X$ with a point-countable base:

1. There is no well-ordering of $X$ with all initial segments closed in $X$.

2. $X \rightarrow(\omega+1)_{2}^{2}$.

3. $X \rightarrow(\omega+1)_{l}^{k}$ for all natural numbers $k, l \geq 1$.

It turns out that the negation of Theorem 14(1) is one of the standard smallness requirements on a space, which in the class of metrisable spaces is equivalent to $\sigma$-discreteness. Thus we have the following definition: 
Definition 15 [6]. A topological space $\langle X, \mathcal{T}\rangle$ is said to be left-separated if there exists a well-ordering $<_{\text {wo }}$ of $X$ so that for each $x \in X,\left\{y \in X: y<_{\text {wo }} x\right\}$ is a closed set.

The proof of the implication from Theorem 14(2) to (1) has some information of interest to us here. To see this assume, that (1) fails and fix a well-ordering $<_{\text {wo }}$ on $X$ with all initial segments closed. So for every $y \in X$, we can fix a closed neighbourhood $U_{y}$ of $y$ which is disjoint from $\left\{x \in X: x<_{\text {wo }} y\right\}$. Define $c:[X]^{2} \rightarrow 2$ by letting $c(x, y)=0$ if and only if $y \in U_{x}$ for all pairs $x, y \in X$ satisfying $x<_{\text {wo }} y$. It is easily checked that subsets $Y$ of $X$ for which $c$ is constant on $[Y]^{2}$ must be discrete. So in particular, $X \rightarrow(\omega+1)_{2}^{2}$, and therefore $X \rightarrow(\mathbb{Q})_{2}^{2}$. In [10], Gerlits and Szentmiklóssy have given an interesting variation of left-separation which is equivalent to it in the class of spaces with a point-countable base. It is condition (1) of the following corollary:

Corollary 16. The following are equivalent for every regular space $X$ with a point-countable base:

1. There is a neighbourhood assignment $U_{x}(x \in X)$ such that for all infinite $Y \subseteq X$ there is $y \in Y$ such that $\left\{x \in Y: y \notin U_{x}\right\}$ is infinite.

2. There is a well-ordering of $X$ with all initial segments closed.

3. $X \rightarrow(\omega+1)_{3}^{2}$.

Proof. The equivalence of (2) and (3) is by Theorem 14. It is clear that (2) implies (1) using a neighbourhood assignment such that $U_{y} \cap\left\{x \in X: x<_{\text {wo }} y\right\}=\emptyset$ for all $y \in X$, where $<_{\text {wo }}$ is a wellordering on $X$ with all initial segments closed. To show that (1) implies (3), consider the colouring $c:[X]^{2} \rightarrow 3$ defined as follows, where $<_{\text {wo }}$ is a fixed well-ordering of $X$ and we assume that the neighbourhood assignment $U_{x}(x \in X)$ witnessing (1) consists of closed neighbourhoods. For $x<$ wo $y$, set $c(x, y)=0$ if $x \notin U_{y}$ and $y \notin U_{x}$; set $c(x, y)=1$ if $x \in U_{y}$; finally, set $c(x, y)=2$ if $x \notin U_{y}$ but $y \in U_{x}$. Note that if $Y \subseteq X$ is such that $c^{\prime \prime}[Y]^{2}=\{1\}$, then $Y$ must be finite or else we would contradict (1). Note also that any $Y \subseteq X$ such that $c^{\prime \prime}[Y]^{2}=\{0\}$ or $c^{\prime \prime}[Y]^{2}=\{2\}$ must be discrete. So the colouring $c$ witnesses (3).

The second author showed in [28] that the existence of a strongly compact cardinal implies that if $X$ is any regular space with a point-countable base, then $X$ is universally meager if and only if it is left-separated. A space $X$ is called universally meager if every continuous function from a Baire space into $X$ must be constant on some nonmeager subset of its domain. This result answered a question of Haydon [11], and shows that in the class of regular spaces with a point-countable base, left-separation coincides with the dual of the well-studied notion of a universally null set. We recall that E. Borel [2] conjectured that the notion of a strong measure-zero set, which is a strengthening of the notion of a universally null set, coincides with the countability requirement on sets of reals - a conjecture which was proved to be consistent by Laver [17] much later.

These surveyed results lead to the following project:

General Problem. Discover the optimal class of regular topological spaces in which the 2-dimensional Ramsey degree of $\mathbb{Q}$ is at most 2 and, more generally, the $k$-dimensional Ramsey degree of $\mathbb{Q}$ is at most $k !(k-1) !$

In this paper, we will address the general problem in dimension 2 for all regular spaces with pointcountable bases. Our main theorem is the following:

Theorem 17. Assume (*). If $X$ is any regular space that is not left-separated and has a point-countable base, then the 2-dimensional Ramsey degree of $\mathbb{Q}$ within $X$ is at most 2.

Corollary 18. Assume that there is either a proper class of Woodin cardinals or one strongly compact cardinal. Then the conclusion of Theorem 17 holds. 
Note that Theorem 3 immediately follows from Theorem 17, because metrisable spaces have pointcountable bases, and they are left-separated if and only if they are $\sigma$-discrete. Furthermore, if $X$ is also a Tychonoff space, then by Theorem 12 the conclusion of Theorem 17 can be improved to say that the 2-dimensional Ramsey degree of $\mathbb{Q}$ in $X$ is exactly equal to 2 . Note that metrisable spaces are Tychonoff.

Our forthcoming paper [19] will treat the General Problem in higher dimensions.

\section{Notation}

Our set-theoretic notation is standard. If $\lambda$ is an infinite cardinal, then $H(\lambda)$ denotes the set of all sets that are hereditarily of cardinality $<\lambda$. The notation $M\langle H(\lambda)$ means that $\langle M, \epsilon\rangle$ is an elementary submodel of the structure $\langle H(\lambda), \in\rangle$. For any $A, \mathcal{P}(A)$ denotes the powerset of $A$ - that is, $\mathcal{P}(A)=\{a: a \subseteq A\}$. For any $A$ and $B, A^{B}$ is the collection of all functions from $B$ to $A$. If $\delta$ is an ordinal, then $A^{<\delta}=\bigcup_{\gamma<\delta} A^{\gamma}$. If $f$ is a function, then $\operatorname{dom}(f)$ denotes the domain of $f$, and if $X \subseteq \operatorname{dom}(f)$, then $f^{\prime \prime} X$ is the image of $X$ under $f$ - that is, $f^{\prime \prime} X=\{f(x): x \in X\}$.

\section{Some preliminaries}

Properties of stationary sets will be used extensively in the proof of the main result. In this section, we will collect together important facts needed in Section 6. Most of this material is standard. We will need to deal only with stationary subsets of $[A]^{<\aleph_{1}}$, for various sets $A$. Other more general notions of stationarity have been considered in the literature. For example, one could talk about stationary subsets of $\mathcal{P}(A)$, for any nonempty set $A$. The interested reader may consult [12] or [16].

Definition 19. Let $A$ be a nonempty set. Then $C \subseteq[A]^{<\aleph_{1}}$ is called a club in $[A]^{<\aleph_{1}}$ if the following two things hold:

1. For any $N \in[A]^{<\aleph_{1}}$, there exists $M \in C$ with $N \subseteq M$.

2. For any $0<\xi<\aleph_{1}$ and any sequence $\left\langle M_{\zeta}: \zeta<\xi\right\rangle$ of elements of $C$, if $\forall \zeta^{\prime} \leq \zeta<\xi\left[M_{\zeta^{\prime}} \subseteq M_{\zeta}\right]$, then $\bigcup_{\zeta<\xi} M_{\zeta} \in C$.

We say that $S \subseteq[A]^{<\aleph_{1}}$ is stationary in $[A]^{<\aleph_{1}}$ if $S \cap C \neq 0$ for every $C \subseteq[A]^{<\aleph_{1}}$ which is a club in $[A]^{<\boldsymbol{\aleph}_{1}}$. And $S \subseteq[A]^{<\boldsymbol{\aleph}_{1}}$ is said to be nonstationary in $[A]^{<\boldsymbol{\aleph}_{1}}$ if it is not stationary in $[A]^{<\boldsymbol{\aleph}_{1}}$.

One of the salient facts about the nonstationary subsets of $[A]^{<\aleph_{1}}$ is that they form a normal $\sigma$-ideal.

Theorem 20 (Jech [12]). Let A be a nonempty set. If $\mathcal{F}$ is any countable family of nonstationary subsets of $[A]^{<\aleph_{1}}$, then $\bigcup \mathcal{F}$ is also a nonstationary subset of $[A]^{<\aleph_{1}}$. If $S$ is a stationary subset of $[A]^{<\aleph_{1}}$, and $F$ is a function such that $\operatorname{dom}(F)=S$ and $\forall M \in S[F(M) \in M]$, then there exists an $m$ so that $\{M \in S: F(M)=m\}$ is a stationary subset of $[A]^{<\aleph_{1}}$.

The last statement of Theorem 20 is usually called the pressing down lemma. The following theorem is a well-known fact about clubs and stationary sets. It governs the behavior of clubs and stationary sets under projections and pullbacks. The reader may refer to [12] or [14] for a proof. This theorem is true even when $\boldsymbol{\aleph}_{1}$ is replaced with an arbitrary regular uncountable cardinal. It is also true for the more general notion of club and stationary set in $\mathcal{P}(X)$. The proof of a version that is applicable to the more general notion of club and stationary set may be found in [16].

Theorem 21. Let $X$ and $Y$ be nonempty sets with $X \subseteq Y$. Then the following hold:

1. If $C \subseteq[X]^{<\aleph_{1}}$ is a club in $[X]^{<\aleph_{1}}$, then

$$
C^{\uparrow Y}=\left\{M \in[Y]^{<\aleph_{1}}: M \cap X \in C\right\}
$$


is a club in $[Y]^{<\aleph_{1}}$.

2. If $S \subseteq[Y]^{<\aleph_{1}}$ is stationary in $[Y]^{<\aleph_{1}}$, then

$$
S_{\downarrow X}=\{M \cap X: M \in S\}
$$

is stationary in $[X]^{<\aleph_{1}}$.

3. If $C \subseteq[Y]^{<\aleph_{1}}$ is a club in $[Y]^{<\aleph_{1}}$, then

$$
C_{\downarrow X}=\{M \cap X: M \in C\} \subseteq[X]^{<\aleph_{1}}
$$

and $C_{\downarrow X}$ contains a club in $[X]^{<\aleph_{1}}$.

4. If $S \subseteq[X]^{<\aleph_{1}}$ is stationary in $[X]^{<N_{1}}$, then

$$
S^{\uparrow Y}=\left\{M \in[Y]^{<\aleph_{1}}: M \cap X \in S\right\}
$$

is stationary in $[Y]^{<\aleph_{1}}$.

Note the asymmetry between (1) and (3) and the symmetry between (2) and (4). We will really only make use of (2) and (4). The relevance of stationary sets to left-separation of topological spaces is taken up next.

Definition 22. Let $\langle X, \mathcal{T}\rangle$ be a topological space. For any $A \subseteq X, \bar{A}$ will denote the closure of $A$. Given a base $\mathcal{B} \subseteq \mathcal{T}$ and a $Y \subseteq X, \mathcal{B}_{Y}$ will denote $\{U \in \mathcal{B}: U \cap Y \neq 0\}$.

Theorem 23 is a deep characterisation of regular left-separated spaces having a point-countable base in terms of nonstationarity of the collection of all countable closed subsets of the space. It first appeared in [6]. Indeed the theorem is also valid for $T_{1}$-spaces. However, all of our spaces are assumed to be regular because we would like to be able to find subspaces homeomorphic to $\mathbb{Q}$ within them.

Theorem 23 (see [6]). If $\langle X, \mathcal{T}\rangle$ is a regular space which has a point-countable base, then $\langle X, \mathcal{T}\rangle$ is not left-separated if and only if $\left\{N \in[X]^{<\aleph_{1}}: \bar{N} \backslash N \neq 0\right\}$ is stationary in $[X]^{<\aleph_{1}}$.

Metrisable spaces that are not $\sigma$-discrete and special stationary Aronszajn lines are both examples of regular non-left-separated spaces with point-countable bases. One of the benefits of a point-countable base is that any countable set which is sufficiently closed under definable operations must contain all the members of the base around any point in its closure. This fact is proved in the next lemma, which will enable us to apply the pressing down lemma:

Lemma 24. Let $\langle X, \mathcal{T}\rangle$ be a topological space with a point-countable base $\mathcal{B} \subseteq \mathcal{T}$. Let $\chi$ be any uncountable regular cardinal and suppose that $M<H(\chi)$, with $|M|=\boldsymbol{\aleph}_{0}$ and $\langle X, \mathcal{T}\rangle, \mathcal{B} \in M$. If $x \in \overline{X \cap M} \backslash M$, then $\mathcal{B}_{\{x\}} \subseteq M$.

Proof. Consider any $U \in \mathcal{B}_{\{x\}}$. It is an open set with $x \in U$, and so $U \cap X \cap M \neq 0$. Choose $y \in U \cap M$. Thus $\{y\} \in M$ and $\mathcal{B}_{\{y\}} \in M$. Since $\mathcal{B}$ is point-countable, $\mathcal{B}_{\{y\}}$ is a countable set. Therefore, $\mathcal{B}_{\{y\}} \subseteq M$. As $U \in \mathcal{B}_{\{y\}}, U \in M$. This shows $\mathcal{B}_{\{x\}} \subseteq M$, as needed.

The countable stationary tower will be our main tool for proving Theorem 17. Building on the groundbreaking work of Foreman, Magidor and Shelah [8], Woodin introduced the stationary tower in [32] and established a wide variety of results in set theory with it. Larson [16] provides an excellent and accessible introduction to the stationary tower and its applications. A more advanced reference is [33]. Towers of ideals, including several variants of the stationary tower, and their associated generic elementary embeddings are studied in [7]. Kanamori [14] provides an introduction to large cardinals. 
Definition 25. Let $\delta$ be a strongly inaccessible cardinal. As usual, $V_{\delta}$ denotes $\{a: \operatorname{rank}(a)<\delta\}$. The countable stationary tower up to $\delta$, denoted $\mathbb{Q}_{<\delta}$, is defined to be the collection of all $\langle A, S\rangle \in V_{\delta}$ such that $A$ is a nonempty set and $S \subseteq[A]^{<\aleph_{1}}$ is stationary in $[A]^{<\aleph_{1}}$. Elements of $\mathbb{Q}<\delta$ will sometimes be called conditions in $\mathbb{Q}<\delta$, or simply conditions.

An ordering on $\mathbb{Q}_{<\delta}$ is defined as follows. For $\langle A, S\rangle,\langle B, T\rangle \in \mathbb{Q}_{<\delta}$, define $\langle B, T\rangle \leq\langle A, S\rangle$ to mean that $B \supseteq A$ and $T \subseteq S^{\uparrow B}$. It is easily checked that $\leq$ is a partial order on $\mathbb{Q}<\delta$. Observe also that for any $\langle B, T\rangle,\langle A, S\rangle \in \mathbb{Q}_{<\delta},\langle B, T\rangle \leq\langle A, S\rangle$ if and only if $B \supseteq A$ and $T_{\downarrow A} \subseteq S$.

If $p \in \mathbb{Q}_{<\delta}$ and $D \subseteq \mathbb{Q}_{<\delta}$, then $D$ is said to be dense below $p$ if for each $\langle A, S\rangle \leq p$, there exists $\langle B, T\rangle \in D$ with $\langle B, T\rangle \leq\langle A, S\rangle$.

Fix a strongly inaccessible cardinal $\delta>\omega$ for the remainder of this section. The following lemma will be useful in conjunction with Lemma 24 and the pressing down lemma:

Lemma 26. Let $\langle X, \mathcal{T}, \mathcal{B}\rangle \in V_{\delta}$ be a regular topological space, where $\mathcal{B} \subseteq \mathcal{T}$ is a point-countable base. If $\langle X, \mathcal{T}\rangle$ is not left-separated, then

$$
p=\left\langle X,\left\{N \in[X]^{<\aleph_{1}}: \bar{N} \backslash N \neq 0\right\}\right\rangle \in \mathbb{Q}_{<\delta} .
$$

Moreover, the collection of all $\langle B, T\rangle \leq p$ with the property that there exists an uncountable regular cardinal $\chi$ such that $B=H(\chi)$ and

$$
\forall M \in T\left[|M|=\aleph_{0} \text { and } M<H(\chi) \text { and }\langle X, \mathcal{T}\rangle, \mathcal{B} \in M \text { and } \overline{X \cap M} \backslash M \neq 0\right]
$$

is dense below p.

Proof. The hypotheses together with Theorem 23 imply that $X$ is a nonempty set, $p \in V_{\delta}$ and $p$ is a condition in $\mathbb{Q}_{<\delta}$. For the second part, let $\langle A, S\rangle \leq p$. Fix an uncountable regular cardinal $\chi$ with $\{A,\langle X, \mathcal{T}\rangle, \mathcal{B}\} \subseteq H(\chi)$ and $H(\chi) \in V_{\delta}$. Let $B=H(\chi)$. Since $A \subseteq B,\left\langle B, S^{\uparrow B}\right\rangle \leq\langle A, S\rangle$. Now it is well known that $C=\left\{M \in[B]^{<\aleph_{1}}: M<H(\chi)\right.$ and $\left.\{A,\langle X, \mathcal{T}\rangle, \mathcal{B}\} \subseteq M\right\}$ is a club in $[B]^{<\aleph_{1}}$. Let $T=C \cap S^{\uparrow B}$. Then $\langle B, T\rangle \leq\left\langle B, S^{\uparrow B}\right\rangle \leq\langle A, S\rangle \leq p$, as required.

Todorcevic [28] defines an ideal $\mathcal{J}_{\omega_{1}}(\delta)$ as follows. Let us say that a set $T \subseteq[\delta]^{<\boldsymbol{\aleph}_{1}}$ depends on a bounded set of coordinates in $\delta$ if there exists a bounded subset $A \subseteq \delta$ with the property that for all $M, M^{\prime} \in[\delta]^{<\aleph_{1}}$, if $M \cap A=M^{\prime} \cap A$, then $M \in T$ if and only if $M^{\prime} \in T$. By $\mathcal{F}_{\omega_{1}}(\delta)$ we denote the collection of all $T \subseteq[\delta]^{<\aleph_{1}}$ that depend on a bounded set of coordinates in $\delta$. For a bounded subset $A \subseteq \delta$ and a function $f: A^{<\omega} \rightarrow A, C_{f}$ denotes $\left\{M \in[\delta]^{<\kappa_{1}}: f^{\prime \prime}\left((M \cap A)^{<\omega}\right) \subseteq M\right\}$. In addition, $\mathcal{J}_{\omega_{1}}(\delta)$ is the collection of all $T \subseteq[\delta]^{<\aleph_{1}}$ for which there exist a bounded $A \subseteq \delta$ and a function $f: A^{<\omega} \rightarrow A$ such that $T \cap C_{f}=\emptyset$. Finally, $\mathcal{B}_{\omega_{1}}(\delta)=\mathcal{F}_{\omega_{1}}(\delta) \backslash \mathcal{J}_{\omega_{1}}(\delta)$. While we will not be working with any of these collections directly, it is worth noting that there is a natural one-to-one correspondence between the members of $\mathbb{Q}_{<\delta}$ and $\mathcal{B}_{\omega_{1}}(\delta)$.

We now consider a version of the Banach-Mazur game played with conditions in $\mathbb{Q}<\delta$. It is also similar to the precipitous game (see [12]).

Definition 27. Define a two-player game $\partial(\delta)$ as follows. Two players, Empty and Nonempty, take turns playing conditions in $\mathbb{Q}_{<\delta}$, with Empty making the first move. When one of the players has played $\left\langle A_{n}, S_{n}\right\rangle \in \mathbb{Q}_{<\delta}$, their opponent is required to play $\left\langle A_{n+1}, S_{n+1}\right\rangle \leq\left\langle A_{n}, S_{n}\right\rangle$. Thus each run of the game produces a sequence

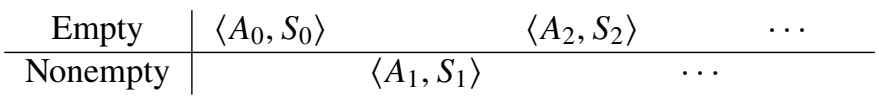

such that for each $n \in \omega,\left\langle A_{2 n}, S_{2 n}\right\rangle$ has been played by Empty, $\left\langle A_{2 n+1}, S_{2 n+1}\right\rangle$ has been played by 
Nonempty and $\left\langle A_{n+1}, S_{n+1}\right\rangle \leq\left\langle A_{n}, S_{n}\right\rangle$. Nonempty wins this particular run of $\partial(\delta)$ if and only if there exists a sequence $\left\langle N_{l}: l \in \omega\right\rangle$ such that $\forall l \in \omega\left[N_{l} \in S_{l}\right]$ and $\forall k \leq l\left[N_{k}=N_{l} \cap A_{k}\right]$.

The following important theorem tells us that if $\delta$ is a suitable large cardinal, then the Empty player does not have a winning strategy in $\partial(\delta)$. It is equivalent to the well-known theorem of Woodin stating that the generic ultrapower of the universe induced by $\mathbb{Q}<\delta$ is well-founded. A version of this theorem for the collection $\mathcal{B}_{\omega_{1}}(\delta)$ was proved by the second author (see Todorcevic [28, Lemma 2.3]). More directly, the proof of [16, Lemma 2.5.6] can be easily adapted to prove Theorem 28.

Theorem 28. If $\delta$ is a Woodin cardinal or a strongly compact cardinal, then Empty does not have a winning strategy in $\mathrm{\partial}(\delta)$.

\section{Main theorem}

Fix, once and for all, an uncountable cardinal $\delta$, which is either Woodin or strongly compact. Fix in addition a regular topological space $\langle X, \mathcal{T}, \mathcal{B}\rangle \in V_{\delta}$, where $\mathcal{B} \subseteq \mathcal{T}$ is a point-countable base and $\langle X, \mathcal{T}\rangle$ is not left-separated. Put $A_{0}=X$ and $S_{0}=\left\{N \in[X]^{<\boldsymbol{\aleph}_{1}}: \bar{N} \backslash N \neq 0\right\}$. Note that $\left\langle A_{0}, S_{0}\right\rangle \in \mathbb{Q}_{<\delta}$.

Definition 29. Fix a function $F: S_{0} \rightarrow X$ such that $F(N) \in \bar{N} \backslash N$, for each $N \in S_{0}$. If $\langle A, S\rangle \leq\left\langle A_{0}, S_{0}\right\rangle$, then for any $M \in S, M \cap A_{0} \in S_{0}$, and we will abuse notation and write $F(M)$ to mean $F\left(M \cap A_{0}\right)$.

We will first prove a sequence of simple lemmas establishing some useful properties of $F$ and the neighbourhoods in $\mathcal{B}$. The first property is that $F$ is 'nowhere constant', meaning that the preimage of every point in $A_{0}$ is nonstationary.

Lemma 30. For any $\langle A, S\rangle \leq\left\langle A_{0}, S_{0}\right\rangle$ and any $x \in A_{0},\{M \in S: F(M)=x\}$ is nonstationary in $[A]^{<\boldsymbol{N}_{1}}$.

Proof. Suppose for a contradiction that $S^{\prime}=\{M \in S: F(M)=x\}$ is stationary in $[A]^{<\aleph_{1}}$. Note that $C=\left\{M \in[A]^{<\aleph_{1}}:\{x\} \subseteq M\right\}$ is a club in $[A]^{<\aleph_{1}}$. Choose $M \in S$ with $x \in M$ and $F(M)=x$. Put $N=M \cap A_{0}$. Then $x \in N$. However, $x=F(M)=F(N) \in \bar{N} \backslash N$. This is a contradiction, completing the proof.

The next property concerns the 'largeness' of neighbourhoods of points in $X$. For any condition in $\mathbb{Q}<\delta$ below $\left\langle A_{0}, S_{0}\right\rangle$, every neighbourhood of almost every point in the image of that condition has large intersection with the same image. This is proved by a simple application of the pressing down lemma.

Definition 31. For each $x \in X$, fix an enumeration $\left\langle U_{x, n}: n \in \omega\right\rangle$ of the set $\{U \in \mathcal{B}: x \in U\}$. For any $\langle A, S\rangle \leq\left\langle A_{0}, S_{0}\right\rangle$, we will say that $M \in S$ is bad if there exists $n \in \omega$ such that $\left\{M^{\prime} \in S: F\left(M^{\prime}\right) \in U_{F(M), n}\right\}$ is nonstationary in $[A]^{<\aleph_{1}}$.

Lemma 32. Suppose $\langle A, S\rangle \leq\left\langle A_{0}, S_{0}\right\rangle$. Then $\{M \in S: M$ is bad $\}$ is nonstationary.

Proof. Write $S_{1}=\{M \in S: M$ is bad $\}$. Assume for a contradiction that $S_{1}$ is stationary in $[A]^{<\aleph_{1}}$. Then $\left\langle A, S_{1}\right\rangle \leq\langle A, S\rangle \leq\left\langle A_{0}, S_{0}\right\rangle$. Applying Lemma 26, there exists $\langle B, T\rangle \leq\left\langle A, S_{1}\right\rangle$ with the property that $B=H(\chi)$, where $\chi$ is an uncountable regular cardinal, and for all $K \in T, K<H(\chi),|K|=\aleph_{0}$ and $\langle X, \mathcal{T}\rangle, \mathcal{B} \in K$. For any $K \in T, M=K \cap A \in S_{1}$, and so $M$ is bad, which means that there exists $n \in \omega$ so that $\left\{M^{\prime} \in S: F\left(M^{\prime}\right) \in U_{F(M), n}\right\}$ is nonstationary in $[A]^{<\aleph_{1}}$. Note that $F(K)=F(M)$ and that $U_{F(K), n} \in K$, because of Lemma 24. Thus for each $K \in T$, we have $U_{K} \in K$ such that $F(K) \in U_{K}$ and $\left\{M^{\prime} \in S: F\left(M^{\prime}\right) \in U_{K}\right\}$ is nonstationary in $[A]^{<\aleph_{1}}$. By the pressing down lemma, there exists $U$ so that

$$
T^{\prime}=\left\{K \in T: U_{K}=U\right\}
$$


is stationary in $[B]^{<\aleph_{1}}$. Since $T^{\prime} \neq \emptyset,\left\{M^{\prime} \in S: F\left(M^{\prime}\right) \in U\right\}$ is nonstationary in $[A]^{<\aleph_{1}}$. On the other hand, $T_{\downarrow A}^{\prime}$ is stationary in $[A]^{<\aleph_{1}}$ and

$$
T_{\downarrow A}^{\prime} \subseteq\left\{M^{\prime} \in S: F\left(M^{\prime}\right) \in U\right\} .
$$

This is a contradiction, which concludes the proof.

Lemma 32 says that for any $\langle A, S\rangle \leq\left\langle A_{0}, S_{0}\right\rangle$, the set $\{M \in S: M$ is not bad $\}$ is almost equal to $S$. Therefore, once all the bad points in $S$ have been thrown away, none of the remaining points can be bad in what is left. So there is no need to repeat the operation of throwing away bad points. This is what Lemma 34 says.

Definition 33. For any $\langle A, S\rangle \leq\left\langle A_{0}, S_{0}\right\rangle$, define $\widetilde{S}=\{M \in S: M$ is not bad $\}$. By Lemma 32, $\langle A, \widetilde{S}\rangle \in \mathbb{Q}_{<\delta}$ and $\langle A, \widetilde{S}\rangle \leq\langle A, S\rangle$.

Lemma 34. Let $\langle A, S\rangle \leq\left\langle A_{0}, S_{0}\right\rangle$. For any $M \in \widetilde{S}$ and any $n \in \omega$,

$$
\left\{M^{\prime} \in \widetilde{S}: F\left(M^{\prime}\right) \in U_{F(M), n}\right\}
$$

is stationary in $[A]^{<\aleph_{1}}$.

Proof. Take any $M \in \widetilde{S}$ and any $n \in \omega$. Then $M$ is not bad in $S$, which means that $\left\{M^{\prime} \in S\right.$ : $\left.F\left(M^{\prime}\right) \in U_{F(M), n}\right\}$ is stationary in $[A]^{<\aleph_{1}}$. Since $S \backslash \widetilde{S}$ is nonstationary in $[A]^{<\aleph_{1}}$, it follows that $\widetilde{S} \cap\left\{M^{\prime} \in S: F\left(M^{\prime}\right) \in U_{F(M), n}\right\}=\left\{M^{\prime} \in \widetilde{S}: F\left(M^{\prime}\right) \in U_{F(M), n}\right\}$ is stationary in $[A]^{<\aleph_{1}}$.

Definition 35. Fix $l \in \omega$ with $l>0$ and fix $c:[X]^{2} \rightarrow l$. Suppose $\langle A, S\rangle \leq\left\langle A_{0}, S_{0}\right\rangle$ and $\langle B, T\rangle \leq\left\langle A_{0}, S_{0}\right\rangle$. For any $i \in l$ and any $M \in S$, define $\mathcal{K}(c, i, M, B, T)$ to be $\left\{M^{\prime} \in T: F(M) \neq F\left(M^{\prime}\right)\right.$ and $\left.c\left(F(M), F\left(M^{\prime}\right)\right)=i\right\}$. We will say that $M$ is $i$-large in $\langle B, T\rangle$ with respect to $c$ if $\mathcal{K}(c, i, M, B, T)$ is stationary in $[B]^{<\aleph_{1}}$.

For any $i, j \in l$, the pair $\langle\langle A, S\rangle,\langle B, T\rangle\rangle$ is said to be $\langle i, j\rangle$-saturated with respect to $c$ if for any $\left\langle A^{\prime}, S^{\prime}\right\rangle \leq\langle A, S\rangle$ and any $\left\langle B^{\prime}, T^{\prime}\right\rangle \leq\langle B, T\rangle$, both of the following hold:

1. $\left\{M \in S^{\prime}: M\right.$ is $i$-large in $\left\langle B^{\prime}, T^{\prime}\right\rangle$ with respect to $\left.c\right\}$ is stationary in $\left[A^{\prime}\right]^{<\aleph_{1}}$.

2. $\left\{K \in T^{\prime}: K\right.$ is $j$-large in $\left\langle A^{\prime}, S^{\prime}\right\rangle$ with respect to $\left.c\right\}$ is stationary in $\left[B^{\prime}\right]^{<\kappa_{1}}$.

As the colouring $c$ will remain fixed until the end of the proof of Theorem 47, we will generally omit the phrase 'with respect to $c$ ' everywhere in what follows.

Intuitively, if a pair $\langle\langle A, S\rangle,\langle B, T\rangle\rangle$ is $\langle i, j\rangle$-saturated, then the colours $i$ and $j$ occur in every rectangle whose sides are conditions below $\langle A, S\rangle$ and $\langle B, T\rangle$ in $\mathbb{Q}<\delta$. More precisely, any rectangle whose base is a condition below $\langle A, S\rangle$ and whose height is a condition below $\langle B, T\rangle$ must contain many vertical columns with a large collection of $i$-coloured points, and also many horizontal rows with a large collection of $j$-coloured points.

Lemma 36. Suppose $\left\langle A^{\prime}, S^{\prime}\right\rangle \leq\langle A, S\rangle \leq\left\langle A_{0}, S_{0}\right\rangle$ and $\left\langle B^{\prime}, T^{\prime}\right\rangle \leq\langle B, T\rangle \leq\left\langle A_{0}, S_{0}\right\rangle$. For any $i \in l$ and any $M \in S^{\prime}, \mathcal{K}(c, i, M, B, T)=\mathcal{K}(c, i, M \cap A, B, T)$. Also, if $M$ is $i$-large in $\left\langle B^{\prime}, T^{\prime}\right\rangle$, then $M$ is $i$-large in $\langle B, T\rangle$. Further, if $M$ is $i$-large in $\left\langle B^{\prime}, T^{\prime}\right\rangle$, then $M \cap A$ is $i$-large in $\langle B, T\rangle$.

Proof. Indeed, $M \cap A \in S$ and $F(M)=F(M \cap A)$, and so

$$
\begin{aligned}
\mathcal{K}(c, i, M, B, T) & =\left\{M^{\prime} \in T: F(M \cap A) \neq F\left(M^{\prime}\right) \text { and } c\left(F(M \cap A), F\left(M^{\prime}\right)\right)=i\right\} \\
& =\mathcal{K}(c, i, M \cap A, B, T) .
\end{aligned}
$$


Moreover, if $M$ is $i$-large in $\left\langle B^{\prime}, T^{\prime}\right\rangle$, then $\mathcal{K}\left(c, i, M, B^{\prime}, T^{\prime}\right)$ is stationary in $\left[B^{\prime}\right]^{<\aleph_{1}}$. Since $\left(\mathcal{K}\left(c, i, M, B^{\prime}, T^{\prime}\right)\right)_{\downarrow B}$ is stationary in $[B]^{<\aleph_{1}}$ and we have that $\left(\mathcal{K}\left(c, i, M, B^{\prime}, T^{\prime}\right)\right)_{\downarrow B} \subseteq$ $\mathcal{K}(c, i, M, B, T) \subseteq T \subseteq[B]^{<\aleph_{1}}$, it follows that $\mathcal{K}(c, i, M, B, T)$ is stationary in $[B]^{<\aleph_{1}}$. Hence $M$ is $i$-large in $\langle B, T\rangle$. Finally, if $M$ is $i$-large in $\left\langle B^{\prime}, T^{\prime}\right\rangle$, then by what we have remarked up to now, $\mathcal{K}(c, i, M, B, T)=\mathcal{K}(c, i, M \cap A, B, T)$ is stationary in $[B]^{<\aleph_{1}}$, and so $M \cap A$ is $i$-large in $\langle B, T\rangle$.

The next lemma expresses the simple fact that for a fixed row or column in any rectangle, there must be a colour that occurs frequently along that row or column:

Lemma 37. Suppose $\langle A, S\rangle \leq\left\langle A_{0}, S_{0}\right\rangle$ and $\langle B, T\rangle \leq\left\langle A_{0}, S_{0}\right\rangle$. For each $M \in S$ and each $K \in T$, there exists $\langle i, j\rangle \in l \times l$ such that $M$ is $i$-large in $\langle B, T\rangle$ and $K$ is $j$-large in $\langle A, S\rangle$.

Proof. Put $x=F(M)$ and $y=F(K)$. By Lemma 30, $T^{\prime}=\left\{K^{\prime} \in T: F\left(K^{\prime}\right) \neq x\right\}$ is stationary in $[B]^{<\aleph_{1}}$ and $S^{\prime}=\left\{M^{\prime} \in S: F\left(M^{\prime}\right) \neq y\right\}$ is stationary in $[A]^{<\aleph_{1}}$. For each $i<l$, let $T_{i}^{\prime}=\left\{K^{\prime} \in\right.$ $\left.T^{\prime}: c\left(F(M), F\left(K^{\prime}\right)\right)=i\right\}$ and let $S_{i}^{\prime}=\left\{M^{\prime} \in S^{\prime}: c\left(F(K), F\left(M^{\prime}\right)\right)=i\right\}$. There must be a pair $\langle i, j\rangle \in l \times l$ such that $T_{i}^{\prime} \subseteq[B]^{<\aleph_{1}}$ is stationary and $S_{j}^{\prime} \subseteq[A]^{<\kappa_{1}}$ is stationary, because $\bigcup_{i<l} T_{i}^{\prime}=T^{\prime}$ and $\bigcup_{i<l} S_{i}^{\prime}=S^{\prime}$. Since $T_{i}^{\prime} \subseteq \mathcal{K}(c, i, M, B, T) \subseteq[B]^{<\aleph_{1}}$ and $S_{j}^{\prime} \subseteq \mathcal{K}(c, j, K, A, S) \subseteq[A]^{<\aleph_{1}}, M$ is $i$-large in $\langle B, T\rangle$ and $K$ is $j$-large in $\langle A, S\rangle$.

It is obvious from the definition that the property of being $\langle i, j\rangle$-saturated is hereditary. We state this as a separate fact because it will be useful, but we will omit the trivial proof.

Lemma 38. Suppose $\left\langle A^{\prime}, S^{\prime}\right\rangle \leq\langle A, S\rangle \leq\left\langle A_{0}, S_{0}\right\rangle$ and $\left\langle B^{\prime}, T^{\prime}\right\rangle \leq\langle B, T\rangle \leq\left\langle A_{0}, S_{0}\right\rangle$. For any $\langle i, j\rangle \in$ $l \times l$, if $\langle\langle A, S\rangle,\langle B, T\rangle\rangle$ is $\langle i, j\rangle$-saturated, then so is $\left\langle\left\langle A^{\prime}, S^{\prime}\right\rangle,\left\langle B^{\prime}, T^{\prime}\right\rangle\right\rangle$.

The next lemma will play an important role in the rest of the proof. It asserts the existence of a single pair of colours $\langle i, j\rangle$ and a condition in $\mathbb{Q}<\delta$ with the property that every condition below it in $\mathbb{Q}_{<\delta}$ can be split into an $\langle i, j\rangle$-saturated pair. The proof is an exhaustion argument.

Lemma 39. There exist $\langle i, j\rangle \in l \times l$ and $\left\langle A_{1}, S_{1}\right\rangle \leq\left\langle A_{0}, S_{0}\right\rangle$ such that for any $\left\langle A_{2}, S_{2}\right\rangle \leq\left\langle A_{1}, S_{1}\right\rangle$, there exist $\langle A, S\rangle \leq\left\langle A_{2}, S_{2}\right\rangle$ and $\langle B, T\rangle \leq\left\langle A_{2}, S_{2}\right\rangle$ such that $\langle\langle A, S\rangle,\langle B, T\rangle\rangle$ is $\langle i, j\rangle$-saturated.

Proof. Since $l>0$, we can enumerate the members of $l \times l$ as $\left\{\left\langle i_{1}, j_{1}\right\rangle, \ldots,\left\langle i_{l^{2}}, j_{l^{2}}\right\rangle\right\}$. Suppose that the statement of the lemma fails. Then there exists a sequence $\left\langle A_{0}, S_{0}\right\rangle \geq\left\langle A_{1}, S_{1}\right\rangle \geq \cdots \geq\left\langle A_{l^{2}}, S_{l^{2}}\right\rangle$ such that for each $1 \leq k \leq l^{2},\left\langle A_{k}, S_{k}\right\rangle$ has the property that for any $\langle A, S\rangle \leq\left\langle A_{k}, S_{k}\right\rangle$ and any $\langle B, T\rangle \leq\left\langle A_{k}, S_{k}\right\rangle,\langle\langle A, S\rangle,\langle B, T\rangle\rangle$ is not $\left\langle i_{k}, j_{k}\right\rangle$-saturated. Next build three sequences

$$
\begin{aligned}
& \left\langle A_{l^{2}}, S_{l^{2}}\right\rangle=\left\langle A_{0}^{\prime}, S_{0}^{\prime}\right\rangle \geq\left\langle A_{1}^{\prime}, S_{1}^{\prime}\right\rangle \geq \cdots \geq\left\langle A_{l^{2}}^{\prime}, S_{l^{2}}^{\prime}\right\rangle, \\
& \left\langle A_{l^{2}}, S_{l^{2}}\right\rangle=\left\langle B_{0}^{\prime}, T_{0}^{\prime}\right\rangle \geq\left\langle B_{1}^{\prime}, T_{1}^{\prime}\right\rangle \geq \cdots \geq\left\langle B_{l^{2}}^{\prime}, T_{l^{2}}^{\prime}\right\rangle \text { and } \\
& \left\langle S_{1}^{*}, T_{1}^{*}\right\rangle, \ldots,\left\langle S_{l^{2}}^{*}, T_{l^{2}}^{*}\right\rangle
\end{aligned}
$$

such that

1. for each $1 \leq k \leq l^{2}, S_{k}^{*} \subseteq S_{k}^{\prime}$ is nonstationary in $\left[A_{k}^{\prime}\right]^{<\aleph_{1}}$ and $T_{k}^{*} \subseteq T_{k}^{\prime}$ is nonstationary in $\left[B_{k}^{\prime}\right]^{<\aleph_{1}}$;

2. for each $1 \leq k \leq l^{2}$,

$$
\begin{aligned}
& \text { either } S_{k}^{*}=\left\{M \in S_{k}^{\prime}: M \text { is } i_{k} \text {-large in }\left\langle B_{k}^{\prime}, T_{k}^{\prime}\right\rangle\right\} \\
& \text { or } T_{k}^{*}=\left\{K \in T_{k}^{\prime}: K \text { is } j_{k} \text {-large in }\left\langle A_{k}^{\prime}, S_{k}^{\prime}\right\rangle\right\} .
\end{aligned}
$$

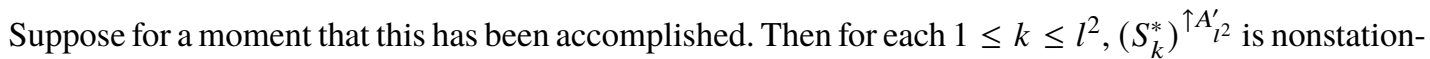

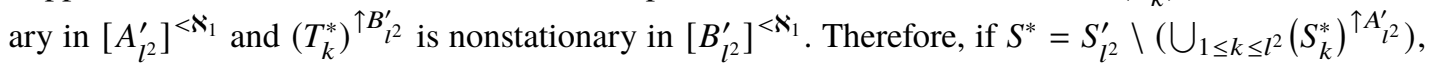




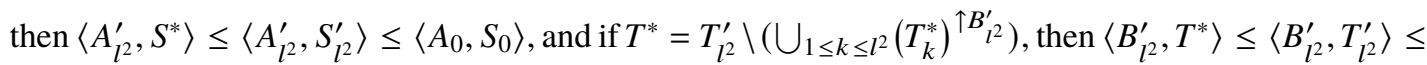
$\left\langle A_{0}, S_{0}\right\rangle$. Choose $M^{*} \in S^{*}$ and $K^{*} \in T^{*}$. Apply Lemma 37 to find $1 \leq k \leq l^{2}$ such that $M^{*}$ is $i_{k}$-large in $\left\langle B_{l^{2}}^{\prime}, T^{*}\right\rangle$ and $K^{*}$ is $j_{k}$-large in $\left\langle A_{l^{2}}^{\prime}, S^{*}\right\rangle$. Note that $M^{*} \cap A_{k}^{\prime} \in S_{k}^{\prime} \backslash S_{k}^{*}$ and $K^{*} \cap B_{k}^{\prime} \in T_{k}^{\prime} \backslash T_{k}^{*}$. By Lemma 36, $M^{*} \cap A_{k}^{\prime}$ is $i_{k}$-large in $\left\langle B_{k}^{\prime}, T_{k}^{\prime}\right\rangle$ and $K^{*} \cap B_{k}^{\prime}$ is $j_{k}$-large in $\left\langle A_{k}^{\prime}, S_{k}^{\prime}\right\rangle$. However, these facts contradict (2), because they imply that $S_{k}^{*} \neq\left\{M \in S_{k}^{\prime}: M\right.$ is $i_{k}$-large in $\left.\left\langle B_{k}^{\prime}, T_{k}^{\prime}\right\rangle\right\}$ and $T_{k}^{*} \neq\left\{K \in T_{k}^{\prime}: K\right.$ is $j_{k}$-large in $\left.\left\langle A_{k}^{\prime}, S_{k}^{\prime}\right\rangle\right\}$.

To construct such sequences, proceed by induction. To start, let $\left\langle A_{0}^{\prime}, S_{0}^{\prime}\right\rangle=\left\langle A_{l^{2}}, S_{l^{2}}\right\rangle=\left\langle B_{0}^{\prime}, T_{0}^{\prime}\right\rangle$. Now suppose that $0 \leq k<k+1 \leq l^{2}$ and that $\left\langle A_{k}^{\prime}, S_{k}^{\prime}\right\rangle \leq\left\langle A_{l^{2}}, S_{l^{2}}\right\rangle$ and $\left\langle B_{k}^{\prime}, T_{k}^{\prime}\right\rangle \leq\left\langle A_{l^{2}}, S_{l^{2}}\right\rangle$ are given. Then $\left\langle A_{k}^{\prime}, S_{k}^{\prime}\right\rangle \leq\left\langle A_{k+1}, S_{k+1}\right\rangle$ and $\left\langle B_{k}^{\prime}, T_{k}^{\prime}\right\rangle \leq\left\langle A_{k+1}, S_{k+1}\right\rangle$. By the choice of $\left\langle A_{k+1}, S_{k+1}\right\rangle$, $\left\langle\left\langle A_{k}^{\prime}, S_{k}^{\prime}\right\rangle,\left\langle B_{k}^{\prime}, T_{k}^{\prime}\right\rangle\right\rangle$ is not $\left\langle i_{k+1}, j_{k+1}\right\rangle$-saturated. Therefore we can find $\left\langle A_{k+1}^{\prime}, S_{k+1}^{\prime}\right\rangle \leq\left\langle A_{k}^{\prime}, S_{k}^{\prime}\right\rangle$ and $\left\langle B_{k+1}^{\prime}, T_{k+1}^{\prime}\right\rangle \leq\left\langle B_{k}^{\prime}, T_{k}^{\prime}\right\rangle$ such that either

$$
\left\{M \in S_{k+1}^{\prime}: M \text { is } i_{k+1} \text {-large in }\left\langle B_{k+1}^{\prime}, T_{k+1}^{\prime}\right\rangle\right\}
$$

is nonstationary in $\left[A_{k+1}^{\prime}\right]^{<\aleph_{1}}$ or

$$
\left\{K \in T_{k+1}^{\prime}: K \text { is } j_{k+1} \text {-large in }\left\langle A_{k+1}^{\prime}, S_{k+1}^{\prime}\right\rangle\right\}
$$

is nonstationary in $\left[B_{k+1}^{\prime}\right]^{<\aleph_{1}}$. If the first alternative happens, then define

$$
S_{k+1}^{*}=\left\{M \in S_{k+1}^{\prime}: M \text { is } i_{k+1} \text {-large in }\left\langle B_{k+1}^{\prime}, T_{k+1}^{\prime}\right\rangle\right\} \subseteq S_{k+1}^{\prime}
$$

and $T_{k+1}^{*}=\emptyset$; if the second alternative occurs, then define $S_{k+1}^{*}=\emptyset$ and

$$
T_{k+1}^{*}=\left\{K \in T_{k+1}^{\prime}: K \text { is } j_{k+1} \text {-large in }\left\langle A_{k+1}^{\prime}, S_{k+1}^{\prime}\right\rangle\right\} \subseteq T_{k+1}^{\prime} .
$$

It is clear that $\left\langle A_{k+1}^{\prime}, S_{k+1}^{\prime}\right\rangle,\left\langle B_{k+1}^{\prime}, T_{k+1}^{\prime}\right\rangle, S_{k+1}^{*}$ and $T_{k+1}^{*}$ are as required. This completes the construction and the proof.

We should point out that in certain special circumstances, it is possible to ensure that $i=j$ in Lemma 39. Suppose for a moment that $X^{2}$ is a Baire space, that $c$ is Baire measurable and that the Kuratowski-Ulam theorem is applicable in every open subset of $X^{2}$. Under these circumstances, $\mathbb{Q}<\delta$ may be replaced everywhere by the coideal of nonmeager subsets of $X$. By Baire measurability, there must be a colour $i$ and open sets $U_{0}, U_{1} \subseteq X$ such that the $i$-coloured points are comeager relative to $U_{0} \times U_{1}$. By Kuratowski-Ulam, almost all the points in almost all vertical sections of $U_{0} \times U_{1}$ must have colour $i$. In fact, under these assumptions, the rest of our proof can be completed using the coideal of nonmeager sets to produce a homeomorphic copy of $\mathbb{Q}$ that is monochromatic in the colour $i$. This should be compared to a theorem of Todorcevic [29] saying that if $c:[\mathbb{Q}]^{2} \rightarrow \mathbb{N}$ is any continuous colouring, where $\mathbb{N}$ is given the discrete topology, then there exists a monochromatic $Y \subseteq \mathbb{Q}$ which is homeomorphic to $\mathbb{Q}$.

The next lemma will only be used in the final construction. It is a simple consequence of the fact that the nonstationary sets form a $\sigma$-ideal.

Lemma 40. Suppose $\mathcal{F} \subseteq \mathbb{Q}_{<\delta}$ is a countable family so that

$$
\forall\langle B, T\rangle \in \mathcal{F}\left[\langle B, T\rangle \leq\left\langle A_{0}, S_{0}\right\rangle\right] .
$$

Suppose $k \in$ l. Let $\langle A, S\rangle \leq\left\langle A_{0}, S_{0}\right\rangle$ have the property that for any $\left\langle A^{\prime}, S^{\prime}\right\rangle \leq\langle A, S\rangle$ and any $\langle B, T\rangle \in \mathcal{F}$, $\left\{M^{\prime} \in S^{\prime}: M^{\prime}\right.$ is $k$-large in $\left.\langle B, T\rangle\right\}$ is stationary in $\left[A^{\prime}\right]^{<\aleph_{1}}$. Then for any $\left\langle A^{\prime}, S^{\prime}\right\rangle \leq\langle A, S\rangle$,

$$
\left\{M^{\prime} \in S^{\prime}: \exists\langle B, T\rangle \in \mathcal{F}\left[M^{\prime} \text { is not } k \text {-large in }\langle B, T\rangle\right]\right\}
$$

is nonstationary in $\left[A^{\prime}\right]^{<\aleph_{1}}$. 
Proof. We argue by contradiction. If there exists an $\left\langle A^{\prime}, S^{\prime}\right\rangle \leq\langle A, S\rangle$ for which the statement of the lemma fails, then there exists a set $S^{\prime \prime} \subseteq S^{\prime}$ which is stationary in $\left[A^{\prime}\right]^{<\aleph_{1}}$ and has the property that for any $M^{\prime} \in S^{\prime \prime}$, there exists $\left\langle B_{M^{\prime}}, T_{M^{\prime}}\right\rangle \in \mathcal{F}$ such that $M^{\prime}$ is not $k$-large in $\left\langle B_{M^{\prime}}, T_{M^{\prime}}\right\rangle$. Since $\mathcal{F}$ is a countable set, it follows that there exists $\langle B, T\rangle \in \mathcal{F}$ so that $S^{*}=\left\{M^{\prime} \in S^{\prime \prime}:\langle B, T\rangle=\left\langle B_{M^{\prime}}, T_{M^{\prime}}\right\rangle\right\}$ is stationary in $\left[A^{\prime}\right]^{<\aleph_{1}}$. Thus $\left\langle A^{\prime}, S^{*}\right\rangle \leq\langle A, S\rangle$, and so by the hypothesis on $\langle A, S\rangle,\left\{M^{\prime} \in S^{*}: M^{\prime}\right.$ is $k$-large in $\left.\langle B, T\rangle\right\}$ is stationary in $\left[A^{\prime}\right]^{<\aleph_{1}}$. In particular, this set is nonempty, which contradicts the choice of $S^{\prime \prime}$, concluding the proof.

In view of Lemma 39, we fix for the remainder of this section pairs $\langle i, j\rangle \in l \times l$ and $\left\langle A_{1}, S_{1}\right\rangle \leq\left\langle A_{0}, S_{0}\right\rangle$ such that for any $\left\langle A_{2}, S_{2}\right\rangle \leq\left\langle A_{1}, S_{1}\right\rangle$, there exist $\langle A, S\rangle \leq\left\langle A_{2}, S_{2}\right\rangle$ and $\langle B, T\rangle \leq\left\langle A_{2}, S_{2}\right\rangle$ such that $\langle\langle A, S\rangle,\langle B, T\rangle\rangle$ is $\langle i, j\rangle$-saturated. We will ensure that all the pairs in the homeomorphic copy of $\mathbb{Q}$ which we are going to construct inside $X$ are coloured either $i$ or $j$.

Lemma 41 is another application of the pressing down lemma. Lemma 42 is proved using Lemmas 41 and 39. Item 2 of Lemma 42 is implied by item 1, but it is stated for emphasis.

Lemma 41. Suppose $\langle A, S\rangle \leq\left\langle A_{0}, S_{0}\right\rangle$. For each $n \in \omega$, there exists $U$ so that $\left\{M^{\prime} \in S: U=U_{F\left(M^{\prime}\right), n}\right\}$ is stationary in $[A]^{<\aleph_{1}}$.

Proof. By Lemma 26, there exists $\langle B, T\rangle \leq\langle A, S\rangle$ with the property that there exists an uncountable regular cardinal $\chi$ such that $B=H(\chi)$ and for each $M \in T,|M|=\aleph_{0}, M<H(\chi)$ and $\langle X, \mathcal{T}\rangle, \mathcal{B} \in M$. Consider any $M \in T$. Then $F(M) \in \overline{M \cap X} \backslash M$. So by Lemma 24, $\mathcal{B}_{\{F(M)\}} \subseteq M$. In particular, $U_{F(M), n} \in M$. Thus by the pressing down lemma there exists $U$ such that

$$
T^{\prime}=\left\{M \in T: U=U_{F(M), n}\right\} \subseteq[B]^{<\aleph_{1}}
$$

is stationary. So $T_{\downarrow A}^{\prime} \subseteq[A]^{<\aleph_{1}}$ is stationary. Since

$$
T_{\downarrow A}^{\prime} \subseteq\left\{M^{\prime} \in S: U=U_{F\left(M^{\prime}\right), n}\right\} \subseteq S \subseteq[A]^{<\aleph_{1}},
$$

$\left\{M^{\prime} \in S: U=U_{F\left(M^{\prime}\right), n}\right\}$ is also stationary in $[A]^{<\aleph_{1}}$.

Lemma 42. Suppose $\langle A, S\rangle \leq\left\langle A_{1}, S_{1}\right\rangle$ and $n \in \omega$. There exist $\left\langle A^{\prime}, S^{\prime}\right\rangle,\left\langle B^{\prime}, T^{\prime}\right\rangle \leq\langle A, \widetilde{S}\rangle$ and there exists $U$ satisfying the following:

1. For all $M^{\prime} \in S^{\prime}, U=U_{F\left(M^{\prime}\right), n}$, and for all $K^{\prime} \in T^{\prime}, U=U_{F\left(K^{\prime}\right), n \text {. }}$.

2. For each $M^{\prime} \in S^{\prime}, F\left(M^{\prime}\right) \in U$, and for each $K^{\prime} \in T^{\prime}, F\left(K^{\prime}\right) \in U$.

3. $\left\langle\left\langle A^{\prime}, S^{\prime}\right\rangle,\left\langle B^{\prime}, T^{\prime}\right\rangle\right\rangle$ is $\langle i, j\rangle$-saturated.

Proof. Since $\langle A, \widetilde{S}\rangle \leq\langle A, S\rangle \leq\left\langle A_{1}, S_{1}\right\rangle \leq\left\langle A_{0}, S_{0}\right\rangle$, Lemma 41 applies and implies that there exists $U$ so that $S^{*}=\left\{M \in \widetilde{S}: U=U_{F(M), n}\right\}$ is stationary in $[A]^{<\aleph_{1}}$. So $\left\langle A, S^{*}\right\rangle \leq\langle A, \widetilde{S}\rangle$, and by Lemma 32, $\left\langle A, \widetilde{\left(S^{*}\right)}\right\rangle \leq\left\langle A, S^{*}\right\rangle$. Choose any $M^{*} \in \widetilde{\left(S^{*}\right)}$. Then $S^{* *}=\left\{M \in S^{*}: F(M) \in U_{F\left(M^{*}\right), n}=U\right\}$ is stationary in $[A]^{<\aleph_{1}}$. Therefore, $\left\langle A, S^{* *}\right\rangle \leq\left\langle A, S^{*}\right\rangle \leq\left\langle A_{1}, S_{1}\right\rangle$, and by the choice of $\left\langle A_{1}, S_{1}\right\rangle$, there exist $\left\langle A^{\prime}, S^{\prime}\right\rangle,\left\langle B^{\prime}, T^{\prime}\right\rangle \leq\left\langle A, S^{* *}\right\rangle$ such that $\left\langle\left\langle A^{\prime}, S^{\prime}\right\rangle,\left\langle B^{\prime}, T^{\prime}\right\rangle\right\rangle$ is $\langle i, j\rangle$-saturated. It is clear that $\left\langle A^{\prime}, S^{\prime}\right\rangle$ and $\left\langle B^{\prime}, T^{\prime}\right\rangle$ are as required.

Definition 43. Suppose $x \in A_{0}$ and $\langle A, S\rangle \leq\left\langle A_{1}, S_{1}\right\rangle$. We will say that $x$ is an $\langle i, j\rangle$-winner in $\langle A, S\rangle$ if there exists $M \in \widetilde{S}$ with $F(M)=x$ and there exists a sequence $\left\langle\left\langle\left\langle A_{x, n}, S_{x, n}\right\rangle,\left\langle B_{x, n}, T_{x, n}\right\rangle\right\rangle: n \in \omega\right\rangle$ satisfying the following conditions:

1. For each $n \in \omega,\left\langle A_{x, n}, S_{x, n}\right\rangle,\left\langle B_{x, n}, T_{x, n}\right\rangle \leq\langle A, \widetilde{S}\rangle$, and

$$
\left\langle A_{x, n+1}, S_{x, n+1}\right\rangle,\left\langle B_{x, n+1}, T_{x, n+1}\right\rangle \leq\left\langle A_{x, n}, \widetilde{S}_{x, n}\right\rangle .
$$


2. For each $n \in \omega$, there exists $M \in \widetilde{S}_{x, n}$ with $F(M)=x$, and moreover, for each $M^{\prime} \in S_{x, n}$, $F\left(M^{\prime}\right) \in U_{x, n}$ and each $K^{\prime} \in T_{x, n}$, we have $F\left(K^{\prime}\right) \in U_{x, n}$.

3. For each $n \in \omega,\left\langle\left\langle A_{x, n}, S_{x, n}\right\rangle,\left\langle B_{x, n}, T_{x, n}\right\rangle\right\rangle$ is $\langle i, j\rangle$-saturated.

4. For each $n \in \omega$, for each $K^{\prime} \in T_{x, n}, F\left(K^{\prime}\right) \neq x$ and $c\left(x, F\left(K^{\prime}\right)\right)=i$.

We would like to point out certain features of Definition 43. Intuitively speaking, the sequence of sets $\left\langle T_{x, n}: n \in \omega\right\rangle$ is converging to the $\langle i, j\rangle$-winner $x$. Moreover, $x$ has colour $i$ with all of the points in $T_{x, n}$ for all $n$, and the pair $\left\langle\left\langle B_{x, k}, T_{x, k}\right\rangle,\left\langle B_{x, n}, T_{x, n}\right\rangle\right\rangle$ is $\langle i, j\rangle$-saturated for all $n<k$. These properties of an $\langle i, j\rangle$-winner are formulated and proved in Lemma 45 . And they are essentially the only properties of an $\langle i, j\rangle$-winner that will be used in the final construction. Thus the condition $\left\langle A_{x, n}, S_{x, n}\right\rangle$ is not directly used at all, though it is the reservoir from which the future $\left\langle B_{x, k}, T_{x, k}\right\rangle$ are drawn. Also, in item 2 of Definition 43, the property that $F(M)=x$ for some $M \in \widetilde{S}_{x, n}$ will not be used, though it is automatically ensured by the proof that $\langle i, j\rangle$-winners exist.

The next lemma is the key to the final construction. It asserts that almost every point in any condition below $\left\langle A_{1}, S_{1}\right\rangle$ is an $\langle i, j\rangle$-winner in that condition. Its proof appeals to Theorem 28, and it is the only place in the proof of Theorem 47 where the assumption that $\delta$ is Woodin or strongly compact is essential.

Lemma 44. For any $\langle A, S\rangle \leq\left\langle A_{1}, S_{1}\right\rangle$,

$\{M \in S: F(M)$ is not an $\langle i, j\rangle$-winner in $\langle A, S\rangle\}$

is nonstationary in $[A]^{<\aleph_{1}}$.

Proof. Suppose not. Then

$$
S^{\prime}=\{M \in \widetilde{S}: F(M) \text { is not an }\langle i, j\rangle \text {-winner in }\langle A, S\rangle\}
$$

is stationary in $[A]^{<\aleph_{1}}$. Thus $\left\langle A, S^{\prime}\right\rangle \leq\langle A, \widetilde{S}\rangle \leq\langle A, S\rangle \leq\left\langle A_{1}, S_{1}\right\rangle$. Applying Lemma 42 with $\left\langle A, S^{\prime}\right\rangle$ in place of $\langle A, S\rangle$, choose $\left\langle C_{0}, R_{0}^{\prime}\right\rangle,\left\langle B_{0}, T_{0}\right\rangle \leq\left\langle A, S^{\prime}\right\rangle$ and $U_{0}$ so that for each $M^{\prime} \in R_{0}^{\prime}, F\left(M^{\prime}\right) \in U_{0}=$ $U_{F\left(M^{\prime}\right), 0}$; for each $K^{\prime} \in T_{0}, F\left(K^{\prime}\right) \in U_{0}=U_{F\left(K^{\prime}\right), 0}$; and $\left\langle\left\langle C_{0}, R_{0}^{\prime}\right\rangle,\left\langle B_{0}, T_{0}\right\rangle\right\rangle$ is $\langle i, j\rangle$-saturated. In particular, $R_{0}=\left\{M^{\prime} \in R_{0}^{\prime}: M^{\prime}\right.$ is $i$-large in $\left.\left\langle B_{0}, T_{0}\right\rangle\right\}$ is stationary in $\left[C_{0}\right]^{<\aleph_{1}}$. Now define a strategy for Empty in $\partial(\delta)$ as follows. Suppose that $\sigma$ is a partial run of $\partial(\delta)$ with $|\sigma|=2 n$ (for some $n \in \omega)$, during which Empty has followed their strategy. If $n=0$, then Empty plays $\left\langle C_{0}, \widetilde{R}_{0}\right\rangle$. If $n>0$, then $\sigma(2 n-1) \leq \sigma(0)=\left\langle C_{0}, \widetilde{R}_{0}\right\rangle \leq\left\langle A_{1}, S_{1}\right\rangle$. Applying Lemma 42 with $\sigma(2 n-1)$ in place of $\langle A, S\rangle$, Empty chooses $\left\langle C_{\sigma}, R_{\sigma}^{\prime}\right\rangle,\left\langle B_{\sigma}, T_{\sigma}\right\rangle \leq \sigma(2 n-1)$ and $U_{n}$ so that for each $M^{\prime} \in R_{\sigma}^{\prime}$, $F\left(M^{\prime}\right) \in U_{n}=U_{F\left(M^{\prime}\right), n}$; for each $K^{\prime} \in T_{\sigma}, F\left(K^{\prime}\right) \in U_{n}=U_{F\left(K^{\prime}\right), n}$; and $\left\langle\left\langle C_{\sigma}, R_{\sigma}^{\prime}\right\rangle,\left\langle B_{\sigma}, T_{\sigma}\right\rangle\right\rangle$ is $\langle i, j\rangle$-saturated. In particular, $R_{\sigma}=\left\{M^{\prime} \in R_{\sigma}^{\prime}: M^{\prime}\right.$ is $i$-large in $\left.\left\langle B_{\sigma}, T_{\sigma}\right\rangle\right\}$ is stationary in $\left[C_{\sigma}\right]^{<\aleph_{1}}$. Empty then plays $\left\langle C_{\sigma}, \widetilde{R}_{\sigma}\right\rangle \leq \sigma(2 n-1)$ as the $2 n$th move of this run. This concludes the definition of a strategy for Empty in $\partial(\delta)$.

Since Empty does not have a winning strategy, there is a complete run of $\partial(\delta)$ in which Empty follows the strategy just defined and loses. Therefore, there exist sequences $\left\langle\left\langle C_{n}, R_{n}\right\rangle: n \in \omega\right\rangle$, $\left\langle\left\langle\left\langle C_{2 n}, R_{2 n}^{\prime}\right\rangle,\left\langle B_{2 n}, T_{2 n}\right\rangle\right\rangle: n \in \omega\right\rangle$ and $\left\langle U_{n}: n \in \omega\right\rangle$ satisfying the following:

1. Nonempty wins the run of $\partial(\delta)$ given by

\begin{tabular}{c|ccccc} 
Empty & $\left\langle C_{0}, \widetilde{R}_{0}\right\rangle$ & & $\left\langle C_{2}, \widetilde{R}_{2}\right\rangle$ & & $\cdots$ \\
\hline Nonempty & & $\left\langle C_{1}, R_{1}\right\rangle$ & & $\left\langle C_{3}, R_{3}\right\rangle$ &
\end{tabular}

2. For each $n \in \omega$, for each $M^{\prime} \in R_{2 n}^{\prime}, F\left(M^{\prime}\right) \in U_{n}=U_{F\left(M^{\prime}\right), n}$; for each $K^{\prime} \in T_{2 n}, F\left(K^{\prime}\right) \in U_{n}=$ $U_{F\left(K^{\prime}\right), n}$; and $\left\langle\left\langle C_{2 n}, R_{2 n}^{\prime}\right\rangle,\left\langle B_{2 n}, T_{2 n}\right\rangle\right\rangle$ is $\langle i, j\rangle$-saturated.

3. For each $n \in \omega, R_{2 n}=\left\{M^{\prime} \in R_{2 n}^{\prime}: M^{\prime}\right.$ is $i$-large in $\left.\left\langle B_{2 n}, T_{2 n}\right\rangle\right\}$ is stationary in $\left[C_{2 n}\right]^{<\aleph_{1}}$.

4. For each $n>0,\left\langle C_{2 n}, R_{2 n}^{\prime}\right\rangle,\left\langle B_{2 n}, T_{2 n}\right\rangle \leq\left\langle C_{2 n-1}, R_{2 n-1}\right\rangle$. 
There is a sequence $\left\langle M_{n}: n \in \omega\right\rangle$ so that

$$
\begin{aligned}
& \forall n \in \omega\left[M_{2 n} \in \widetilde{R}_{2 n} \text { and } M_{2 n+1} \in R_{2 n+1}\right] \text { and } \\
& \forall n \leq k<\omega\left[M_{n}=M_{k} \cap C_{n}\right],
\end{aligned}
$$

because Nonempty wins. Define $x=F\left(M_{0}\right)$. Note that for any $n>0, F\left(M_{2 n}\right)=F\left(M_{0}\right)=x$. Furthermore, $M_{0} \cap A \in S^{\prime}$, which means that $M_{0} \cap A \in \widetilde{S}$ and $x=F\left(M_{0} \cap A\right)$ is not an $\langle i, j\rangle$-winner in $\langle A, S\rangle$. We will get a contradiction by showing that $x$ is an $\langle i, j\rangle$-winner in $\langle A, S\rangle$.

First note that if we let $M=M_{0} \cap A$, then $M \in \widetilde{S}$ and $x=F(M)$. Now define a sequence $\left\langle\left\langle\left\langle A_{x, n}, S_{x, n}\right\rangle,\left\langle B_{x, n}, T_{x, n}\right\rangle\right\rangle: n \in \omega\right\rangle$ as follows. Fix $n \in \omega$ and define $\left\langle A_{x, n}, S_{x, n}\right\rangle=\left\langle C_{2 n}, R_{2 n}\right\rangle$. Note that $M_{2 n} \in R_{2 n}$, whence $M_{2 n} \in R_{2 n}^{\prime}$ and $M_{2 n}$ is $i$-large in $\left\langle B_{2 n}, T_{2 n}\right\rangle$, which means that

$$
T_{x, n}=\left\{K^{\prime} \in T_{2 n}: F\left(M_{2 n}\right) \neq F\left(K^{\prime}\right) \text { and } c\left(F\left(M_{2 n}\right), F\left(K^{\prime}\right)\right)=i\right\}
$$

is stationary in $\left[B_{2 n}\right]^{<\aleph_{1}}$. Defining $B_{x, n}=B_{2 n}$, we have that $\left\langle B_{x, n}, T_{x, n}\right\rangle=\left\langle B_{2 n}, T_{x, n}\right\rangle \leq\left\langle B_{2 n}, T_{2 n}\right\rangle$. Moreover, by the definition of $T_{x, n}$, for any $K^{\prime} \in T_{x, n}, x \neq F\left(K^{\prime}\right)$ and $c\left(x, F\left(K^{\prime}\right)\right)=i$, which is what item 4 of Definition 43 says. Also, $\left\langle\left\langle C_{2 n}, R_{2 n}^{\prime}\right\rangle,\left\langle B_{2 n}, T_{2 n}\right\rangle\right\rangle$ is $\langle i, j\rangle$-saturated, $\left\langle A_{x, n}, S_{x, n}\right\rangle=\left\langle C_{2 n}, R_{2 n}\right\rangle \leq$ $\left\langle C_{2 n}, R_{2 n}^{\prime}\right\rangle$ and $\left\langle B_{x, n}, T_{x, n}\right\rangle \leq\left\langle B_{2 n}, T_{2 n}\right\rangle$, which implies that

$$
\left\langle\left\langle A_{x, n}, S_{x, n}\right\rangle,\left\langle B_{x, n}, T_{x, n}\right\rangle\right\rangle
$$

is $\langle i, j\rangle$-saturated, satisfying item 3 of Definition 43. Next, note that $M_{2 n} \in \widetilde{R}_{2 n}=\widetilde{S}_{x, n}$ and $F\left(M_{2 n}\right)=x$. Note also that since $M_{2 n} \in R_{2 n}^{\prime}, U_{n}=U_{F\left(M_{2 n}\right), n}=U_{x, n}$. Moreover, for any $M^{\prime} \in S_{x, n}, F\left(M^{\prime}\right) \in U_{n}=$ $U_{x, n}$, and for any $K^{\prime} \in T_{x, n}, F\left(K^{\prime}\right) \in U_{n}=U_{x, n}$. Hence item 2 of Definition 43 is satisfied. Furthermore, if $n=0$, then $\left\langle A_{x, n}, S_{x, n}\right\rangle \leq\left\langle C_{2 n}, R_{2 n}^{\prime}\right\rangle=\left\langle C_{0}, R_{0}^{\prime}\right\rangle \leq\langle A, \widetilde{S}\rangle$ and $\left\langle B_{x, n}, T_{x, n}\right\rangle \leq\left\langle B_{2 n}, T_{2 n}\right\rangle=$ $\left\langle B_{0}, T_{0}\right\rangle \leq\left\langle A, S^{\prime}\right\rangle \leq\langle A, \widetilde{S}\rangle$. If $n>0$, then $\left\langle C_{2 n}, R_{2 n}^{\prime}\right\rangle,\left\langle B_{2 n}, T_{2 n}\right\rangle \leq\left\langle C_{2 n-1}, R_{2 n-1}\right\rangle \leq\left\langle C_{0}, \widetilde{R}_{0}\right\rangle \leq$ $\langle A, \widetilde{S}\rangle$, and so $\left\langle A_{x, n}, S_{x, n}\right\rangle \leq\left\langle C_{2 n}, R_{2 n}^{\prime}\right\rangle \leq\langle A, \widetilde{S}\rangle$ and $\left\langle B_{x, n}, T_{x, n}\right\rangle \leq\left\langle B_{2 n}, T_{2 n}\right\rangle \leq\langle A, \widetilde{S}\rangle$. Thus $\left\langle A_{x, n}, S_{x, n}\right\rangle,\left\langle B_{x, n}, T_{x, n}\right\rangle \leq\langle A, \widetilde{S}\rangle$ always holds. Finally, we have $\left\langle A_{x, n+1}, S_{x, n+1}\right\rangle \leq\left\langle C_{2 n+2}, R_{2 n+2}^{\prime}\right\rangle \leq$ $\left\langle C_{2 n+1}, R_{2 n+1}\right\rangle \leq\left\langle A_{x, n}, \widetilde{S}_{x, n}\right\rangle$ and

$$
\left\langle B_{x, n+1}, T_{x, n+1}\right\rangle \leq\left\langle B_{2 n+2}, T_{2 n+2}\right\rangle \leq\left\langle C_{2 n+1}, R_{2 n+1}\right\rangle \leq\left\langle A_{x, n}, \widetilde{S}_{x, n}\right\rangle
$$

Thus $\left\langle A_{x, n+1}, S_{x, n+1}\right\rangle,\left\langle B_{x, n+1}, T_{x, n+1}\right\rangle \leq\left\langle A_{x, n}, \widetilde{S}_{x, n}\right\rangle$ holds, and so item 1 of Definition 43 holds.

This concludes the verification that $x$ is an $\langle i, j\rangle$-winner in $\langle A, S\rangle$. Since this yields a contradiction, the proof is complete.

Lemma 45. Suppose $x \in A_{0}$ and $\langle A, S\rangle \leq\left\langle A_{1}, S_{1}\right\rangle$. If $x$ is an $\langle i, j\rangle$-winner in $\langle A, S\rangle$, then there exists a sequence $\left\langle\left\langle B_{x, n}, T_{x, n}\right\rangle: n \in \omega\right\rangle$ such that the following hold for each $n \in \omega$ :

1. $\left\langle B_{x, n}, T_{x, n}\right\rangle \leq\langle A, S\rangle$.

2. For each $K^{\prime} \in T_{x, n}, F\left(K^{\prime}\right) \in U_{x, n}$.

3. For any $n<k<\omega,\left\langle\left\langle B_{x, k}, T_{x, k}\right\rangle,\left\langle B_{x, n}, T_{x, n}\right\rangle\right\rangle$ is $\langle i, j\rangle$-saturated.

4. For each $K^{\prime} \in T_{x, n}, F\left(K^{\prime}\right) \neq x$ and $c\left(x, F\left(K^{\prime}\right)\right)=i$.

Proof. By the definition of an $\langle i, j\rangle$-winner in $\langle A, S\rangle$, there exists a sequence

$$
\left\langle\left\langle\left\langle A_{x, n}, S_{x, n}\right\rangle,\left\langle B_{x, n}, T_{x, n}\right\rangle\right\rangle: n \in \omega\right\rangle
$$

satisfying items 1-4 of Definition 43. We argue that $\left\langle\left\langle B_{x, n}, T_{x, n}\right\rangle: n \in \omega\right\rangle$ has the required properties. Indeed, from item 1 of Definition $43,\left\langle B_{x, n}, T_{x, n}\right\rangle \leq\langle A, \widetilde{S}\rangle \leq\langle A, S\rangle$ for each $n \in \omega$. Next, items 2 and 4 of this lemma follow from items 2 and 4 of Definition 43 , respectively. Finally, for any 
$n \in \omega$ and any $n<k<\omega,\left\langle B_{x, k}, T_{x, k}\right\rangle \leq\left\langle A_{x, n}, S_{x, n}\right\rangle$ by item 1 of Definition 43. If $n \in \omega$, then $\left\langle\left\langle A_{x, n}, S_{x, n}\right\rangle,\left\langle B_{x, n}, T_{x, n}\right\rangle\right\rangle$ is $\langle i, j\rangle$-saturated, whence for any $n<k<\omega,\left\langle\left\langle B_{x, k}, T_{x, k}\right\rangle,\left\langle B_{x, n}, T_{x, n}\right\rangle\right\rangle$ is $\langle i, j\rangle$-saturated.

Definition 46. If $P \subseteq \omega^{<\omega}$ is a (downwards closed) subtree, we say that $\sigma$ is a leaf node of $P$ if $\sigma \in P$ but there is no $m \in \omega$ for which $\sigma^{\frown}\langle m\rangle \in P$. The expression $L(P)$ will denote the collection of all leaf nodes of $P$, and $N(P)$ will denote $P \backslash L(P)$. Thus $P=L(P) \cup N(P)$.

If $\sigma, \tau \in \omega^{<\omega}$ are incomparable, then

$$
\Delta(\sigma, \tau)=\min \{m \in \operatorname{dom}(\sigma) \cap \operatorname{dom}(\tau): \sigma(m) \neq \tau(m)\} .
$$

We say $\sigma<_{\text {lex }} \tau$ if $\sigma$ and $\tau$ are incomparable and $\sigma(\Delta(\sigma, \tau))<\tau(\Delta(\sigma, \tau))$.

We are now ready to prove the main theorem. We will organise the construction of the homeomorphic copy of $\mathbb{Q}$ by associating every node of the tree $\omega^{<\omega}$ to a point in the copy. This makes certain features of the construction easier to visualise. For instance, the points associated to the successors of a node converge to the point associated to that node. Since the construction is inductive, the homeomorphic copy of $\mathbb{Q}$ is naturally well-ordered by the order in which the points are chosen. Our scheme explicitly displays the interplay between this well-ordering and the lexicographic ordering of the tree, as well as the correspondence between this interplay and the colours $i$ and $j$. Of course, we know from Sierpiński's example that such a close correspondence is unavoidable. The sequence of trees $\left\langle P_{m}: m \in \omega\right\rangle$ in the proof of Theorem 47 serves as a bookkeeping device ensuring that once a point has been chosen, all of its neighbourhoods are eventually considered and met.

Theorem 47. There is a nonempty countable $Y \subseteq X$ such that $Y$ is dense in itself and $c^{\prime \prime}[Y]^{2} \subseteq\{i, j\}$.

Proof. We may choose a sequence $\left\langle P_{m}: m \in \omega\right\rangle$ satisfying the following conditions:

1. For each $m \in \omega, P_{m} \subseteq \omega^{<\omega}$ is a nonempty subtree of finite height.

2. For each $m \in \omega$, there exists $\sigma_{m} \in L\left(P_{m}\right)$ such that

$$
P_{m+1}=P_{m} \cup\left\{\left(\sigma_{m}\right)^{\frown}\langle n\rangle: n \in \omega\right\} .
$$

3. $P_{0}=\{\emptyset\}$ and $\omega^{<\omega}=\bigcup_{n \in \omega} P_{n}$.

It is clear that for each $m \in \omega, L\left(P_{m+1}\right)=\left(L\left(P_{m}\right) \backslash\left\{\sigma_{m}\right\}\right) \cup\left\{\left(\sigma_{m}\right) \frown\langle n\rangle: n \in \omega\right\}$ and $N\left(P_{m+1}\right)=$ $N\left(P_{m}\right) \cup\left\{\sigma_{m}\right\}$. Also, if $m<m^{\prime}<\omega$, then $\sigma_{m} \neq \sigma_{m^{\prime}}$ and $\sigma_{m} \in N\left(P_{m^{\prime}}\right)$. Finally, observe that for each $\sigma \in \omega^{<\omega}$, there exists $m \in \omega$ with $\sigma=\sigma_{m}$, and $m+1$ is the minimal $m^{*} \in \omega$ with $\sigma \in N\left(P_{m^{*}}\right)$. We will construct two sequences $\left\langle x_{m+1}: m \in \omega\right\rangle$ and $\left\langle F_{m}: m \in \omega\right\rangle$ such that the following conditions hold at each $m \in \omega$ :

4. $x_{m+1} \in X$ and $F_{m}: L\left(P_{m}\right) \rightarrow \mathbb{Q}_{<\delta}$; for a $\sigma \in L\left(P_{m}\right)$, we will write $\left\langle B_{m, \sigma}, T_{m, \sigma}\right\rangle$ instead of $F_{m}(\sigma)$.

5. For each $\sigma \in L\left(P_{m}\right),\left\langle B_{m, \sigma}, T_{m, \sigma}\right\rangle \leq\left\langle A_{1}, S_{1}\right\rangle$, and furthermore, for each $m^{\prime} \leq m$ and each $\sigma \in L\left(P_{m^{\prime}}\right) \cap L\left(P_{m}\right), T_{m, \sigma} \subseteq T_{m^{\prime}, \sigma}$.

6. For each $m^{\prime}<m$ and each $\sigma \in L\left(P_{m}\right)$, if $\sigma_{m^{\prime}} \subsetneq \sigma$, then for each $K \in T_{m, \sigma}, F(K) \neq x_{m^{\prime}+1}$ and $c\left(F(K), x_{m^{\prime}+1}\right)=i$; if $\sigma_{m^{\prime}}<_{\text {lex }} \sigma$, then for each $K \in T_{m, \sigma}, F(K) \neq x_{m^{\prime}+1}$ and $c\left(F(K), x_{m^{\prime}+1}\right)=j$; if $\sigma<_{\operatorname{lex}} \sigma_{m^{\prime}}$, then for each $K \in T_{m, \sigma}, F(K) \neq x_{m^{\prime}+1}$ and $c\left(F(K), x_{m^{\prime}+1}\right)=i$.

7. For any $\sigma, \tau \in L\left(P_{m}\right)$, if $\sigma<_{\text {lex }} \tau$, then $\left\langle\left\langle B_{m, \tau}, T_{m, \tau}\right\rangle,\left\langle B_{m, \sigma}, T_{m, \sigma}\right\rangle\right\rangle$ is $\langle i, j\rangle$-saturated.

8. There exists $K \in T_{m, \sigma_{m}}$ so that $x_{m+1}=F(K)$.

9. For each $\left.\left.n \in \omega,\left\langle B_{(m+1),\left(\left(\sigma_{m}\right)\right.}-\langle n\rangle\right), T_{(m+1),\left(\left(\sigma_{m}\right)\right.}-\langle n\rangle\right)\right\rangle \leq\left\langle B_{m, \sigma_{m}}, T_{m, \sigma_{m}}\right\rangle$, and furthermore, for each $\left.K \in T_{(m+1),\left(\left(\sigma_{m}\right)\right.}-\langle n\rangle\right), F(K) \in U_{x_{m+1}, n}$.

Suppose for a moment that these two sequences can be built. Define $Y=\left\{x_{m+1}: m \in \omega\right\}$. Clearly $Y \subseteq X$ and $Y$ is countable and nonempty. We first verify that $Y$ is dense in itself. Indeed, fix $m, n \in \omega$. 
We must find some $m^{\prime} \in \omega$ for which $x_{m^{\prime}+1} \in U_{x_{m+1}, n}$ and $x_{m^{\prime}+1} \neq x_{m+1}$. Put $\tau=\left(\sigma_{m}\right)^{-}\langle n\rangle$. Then $\tau \in L\left(P_{m+1}\right)$. Let $m^{\prime} \in \omega$ be so that $\tau=\sigma_{m^{\prime}}$. It is easy to see that $m+1 \leq m^{\prime}$. By item 9 , for each $K \in T_{m+1, \tau}, F(K) \in U_{x_{m+1}, n}$. By item 6 applied to $m<m^{\prime}$ and $\tau \in L\left(P_{m^{\prime}}\right)$, since $\sigma_{m} \subsetneq \tau$, we have that for each $K \in T_{m^{\prime}, \tau}, F(K) \neq x_{m+1}$. By item 5 applied to $m+1 \leq m^{\prime}$ and $\tau \in L\left(P_{m+1}\right) \cap L\left(P_{m^{\prime}}\right)$, we have that $T_{m^{\prime}, \tau} \subseteq T_{m+1, \tau}$. Finally, by item 8 applied to $m^{\prime}$, we have that there exists $K \in T_{m^{\prime}, \tau}$ so that $x_{m^{\prime}+1}=F(K)$. Thus $x_{m^{\prime}+1}=F(K) \neq x_{m+1}$. Also, $K \in T_{m+1, \tau}$, whence $x_{m^{\prime}+1}=F(K) \in U_{x_{m+1}, n}$, as needed. This verifies that $Y$ is dense in itself.

We next check that $c^{\prime \prime}[Y]^{2} \subseteq\{i, j\}$. Consider any $m^{\prime}<m<\omega$. We will verify that $x_{m^{\prime}+1} \neq x_{m+1}$ and that $c\left(x_{m^{\prime}+1}, x_{m+1}\right) \in\{i, j\}$. Apply item 8 to find $K \in T_{m, \sigma_{m}}$ so that $x_{m+1}=F(K)$. We see that $\sigma_{m^{\prime}} \neq \sigma_{m}$, that $\sigma_{m^{\prime}} \in N\left(P_{m}\right)$ and that $\sigma_{m} \in L\left(P_{m}\right)$. In particular, we cannot have $\sigma_{m} \subseteq \sigma_{m^{\prime}}$. Hence by item 6, we have the following three possibilities: if $\sigma_{m^{\prime}} \subsetneq \sigma_{m}$, then $x_{m+1} \neq x_{m^{\prime}+1}$ and $c\left(x_{m+1}, x_{m^{\prime}+1}\right)=i$; if $\sigma_{m^{\prime}}<_{\text {lex }} \sigma_{m}$, then $x_{m+1} \neq x_{m^{\prime}+1}$ and $c\left(x_{m+1}, x_{m^{\prime}+1}\right)=j$; if $\sigma_{m}<_{\text {lex }} \sigma_{m^{\prime}}$, then $x_{m+1} \neq x_{m^{\prime}+1}$ and $c\left(x_{m+1}, x_{m^{\prime}+1}\right)=i$. This is as required.

To finish the proof, it suffices to construct sequences $\left\langle x_{m+1}: m \in \omega\right\rangle$ and $\left\langle F_{m}: m \in \omega\right\rangle$ satisfying items 4-9. We do this by induction. So fix $m^{*} \in \omega$ and assume that $\left\langle x_{m^{\prime}+1}: m^{\prime}<m^{\prime}+1<m^{*}\right\rangle$ and $\left\langle F_{m^{\prime}}: m^{\prime}<m^{*}\right\rangle$ have been defined. We will define $F_{m^{*}}$, and if $m^{*} \neq 0$, then also $x_{m^{*}}$. Since $L\left(P_{0}\right)=P_{0}=\{\emptyset\}$, when $m^{*}=0$ we only need to ensure that $\left\langle B_{0, \emptyset}, T_{0, \emptyset}\right\rangle$ is defined and that it is below $\left\langle A_{1}, S_{1}\right\rangle$. So we define $\left\langle B_{0, \emptyset}, T_{0, \emptyset}\right\rangle=\left\langle A_{1}, S_{1}\right\rangle$. Now suppose that $m^{*}=m+1$, for some $m \in \omega$. Note that since $\sigma_{m} \in L\left(P_{m}\right)$, every $\sigma \in L\left(P_{m}\right) \backslash\left\{\sigma_{m}\right\}$ is incomparable to $\sigma_{m}$. Therefore, $L\left(P_{m}\right) \backslash\left\{\sigma_{m}\right\}=\mathcal{G}_{0} \cup \mathcal{G}_{1}$, where $\mathcal{G}_{0}=\left\{\sigma \in L\left(P_{m}\right): \sigma_{m}<_{\text {lex }} \sigma\right\}$ and $\mathcal{G}_{1}=\left\{\sigma \in L\left(P_{m}\right): \sigma<_{\text {lex }} \sigma_{m}\right\}$. Applying Lemma 40, we conclude that

$$
\left\{K^{\prime} \in T_{m, \sigma_{m}}: \exists \sigma \in \mathcal{G}_{0}\left[K^{\prime} \text { is not } j \text {-large in }\left\langle B_{m, \sigma}, T_{m, \sigma}\right\rangle\right]\right\}
$$

is nonstationary in $\left[B_{m, \sigma_{m}}\right]^{<\aleph_{1}}$ and also that

$$
\left\{K^{\prime} \in T_{m, \sigma_{m}}: \exists \sigma \in \mathcal{G}_{1}\left[K^{\prime} \text { is not } i \text {-large in }\left\langle B_{m, \sigma}, T_{m, \sigma}\right\rangle\right]\right\}
$$

is nonstationary in $\left[B_{m, \sigma_{m}}\right]^{<\aleph_{1}}$. Further, Lemma 44 tells us that

$$
\left\{K^{\prime} \in T_{m, \sigma_{m}}: F\left(K^{\prime}\right) \text { is not an }\langle i, j\rangle \text {-winner in }\left\langle B_{m, \sigma_{m}}, T_{m, \sigma_{m}}\right\rangle\right\}
$$

is nonstationary in $\left[B_{m, \sigma_{m}}\right]^{<\aleph_{1}}$. Therefore, we may choose $K^{\prime} \in T_{m, \sigma_{m}}$ such that the following things are satisfied: $\forall \sigma \in \mathcal{G}_{1}\left[K^{\prime}\right.$ is $i$-large in $\left.\left\langle B_{m, \sigma}, T_{m, \sigma}\right\rangle\right], \forall \sigma \in \mathcal{G}_{0}\left[K^{\prime}\right.$ is $j$-large in $\left.\left\langle B_{m, \sigma}, T_{m, \sigma}\right\rangle\right]$ and $F\left(K^{\prime}\right)$ is an $\langle i, j\rangle$-winner in $\left\langle B_{m, \sigma_{m}}, T_{m, \sigma_{m}}\right\rangle$. Define $x_{m+1}=F\left(K^{\prime}\right)=F\left(K^{\prime} \cap A_{0}\right) \in X$. By Lemma 45, there exists a sequence $\left\langle\left\langle B_{(m+1),\left(\left(\sigma_{m}\right)\right.}{ }_{\langle n\rangle)}, T_{(m+1),\left(\left(\sigma_{m}\right)^{-}\langle n\rangle\right)}\right\rangle: n \in \omega\right\rangle$ such that the following hold for each $n \in \omega$ :

10. $\left.\left.\left\langle B_{(m+1),\left(\left(\sigma_{m}\right)\right.}-\langle n\rangle\right), T_{(m+1),\left(\left(\sigma_{m}\right)\right.}-\langle n\rangle\right)\right\rangle \leq\left\langle B_{m, \sigma_{m}}, T_{m, \sigma_{m}}\right\rangle$.

11. For each $\left.K \in T_{(m+1),\left(\left(\sigma_{m}\right)\right.}-\langle n\rangle\right), F(K) \in U_{x_{m+1}, n}$.

12. For any $n<k<\omega$,

$$
\left\langle\left\langle B_{(m+1),\left(\left(\sigma_{m}\right)^{-}\langle k\rangle\right)}, T_{(m+1),\left(\left(\sigma_{m}\right)^{-}\langle k\rangle\right)}\right\rangle,\left\langle B_{(m+1),\left(\left(\sigma_{m}\right)^{-}\langle n\rangle\right)}, T_{(m+1),\left(\left(\sigma_{m}\right)^{-}\langle n\rangle\right)}\right\rangle\right\rangle
$$

is $\langle i, j\rangle$-saturated.

13. For each $K \in T_{(m+1),\left(\left(\sigma_{m}\right)^{-}\langle n\rangle\right)}, F(K) \neq x_{m+1}$ and $c\left(x_{m+1}, F(K)\right)=i$.

For each $\sigma \in L\left(P_{m}\right) \backslash\left\{\sigma_{m}\right\}$, if $\sigma \in \mathcal{G}_{0}$, then define $B_{m+1, \sigma}=B_{m, \sigma}$ and

$$
T_{m+1, \sigma}=\left\{K \in T_{m, \sigma}: F\left(K^{\prime}\right) \neq F(K) \text { and } c\left(F\left(K^{\prime}\right), F(K)\right)=j\right\},
$$


which is a stationary subset of $\left[B_{m, \sigma}\right]^{<\aleph_{1}}$. If $\sigma \in \mathcal{G}_{1}$, then set $B_{m+1, \sigma}=B_{m, \sigma}$ and

$$
T_{m+1, \sigma}=\left\{K \in T_{m, \sigma}: F\left(K^{\prime}\right) \neq F(K) \text { and } c\left(F\left(K^{\prime}\right), F(K)\right)=i\right\},
$$

which is a stationary subset of $\left[B_{m, \sigma}\right]^{<\aleph_{1}}$. Note that for all $\sigma \in L\left(P_{m}\right) \backslash\left\{\sigma_{m}\right\},\left\langle B_{m+1, \sigma}, T_{m+1, \sigma}\right\rangle \leq$ $\left\langle B_{m, \sigma}, T_{m, \sigma}\right\rangle$. This finishes the definition of $F_{m+1}$ and $x_{m+1}$. It is simple to verify items $4,5,8$ and 9. We will go through the verification of items 6 and 7.

To check item 7, fix any $\sigma, \tau \in L\left(P_{m+1}\right)$ and suppose that $\sigma<_{\operatorname{lex}} \tau$. If $\sigma, \tau \in L\left(P_{m}\right) \backslash\left\{\sigma_{m}\right\}$, then the induction hypothesis applies and implies that

$$
\left\langle\left\langle B_{m, \tau}, T_{m, \tau}\right\rangle,\left\langle B_{m, \sigma}, T_{m, \sigma}\right\rangle\right\rangle
$$

is $\langle i, j\rangle$-saturated. Since we have $\left\langle B_{m+1, \tau}, T_{m+1, \tau}\right\rangle \leq\left\langle B_{m, \tau}, T_{m, \tau}\right\rangle$ and

$$
\left\langle B_{m+1, \sigma}, T_{m+1, \sigma}\right\rangle \leq\left\langle B_{m, \sigma}, T_{m, \sigma}\right\rangle
$$

it follows that $\left\langle\left\langle B_{m+1, \tau}, T_{m+1, \tau}\right\rangle,\left\langle B_{m+1, \sigma}, T_{m+1, \sigma}\right\rangle\right\rangle$ is $\langle i, j\rangle$-saturated. Next, if $\sigma=\left(\sigma_{m}\right)^{-}\langle n\rangle$ and $\tau=\left(\sigma_{m}\right)-\langle k\rangle$ for some $n, k \in \omega$, then $n<k$, and by item $12,\left\langle\left\langle B_{m+1, \tau}, T_{m+1, \tau}\right\rangle,\left\langle B_{m+1, \sigma}, T_{m+1, \sigma}\right\rangle\right\rangle$ is $\langle i, j\rangle$-saturated. Now suppose that

$$
\sigma \in L\left(P_{m}\right) \backslash\left\{\sigma_{m}\right\}
$$

and that $\tau=\left(\sigma_{m}\right)^{\frown}\langle n\rangle$ for some $n \in \omega$. Then $\sigma<_{\text {lex }} \sigma_{m}$, and since $\sigma, \sigma_{m} \in L\left(P_{m}\right)$, the induction hypothesis applies and implies that $\left\langle\left\langle B_{m, \sigma_{m}}, T_{m, \sigma_{m}}\right\rangle,\left\langle B_{m, \sigma}, T_{m, \sigma}\right\rangle\right\rangle$ is $\langle i, j\rangle$-saturated. Since we know that $\left\langle B_{m+1, \sigma}, T_{m+1, \sigma}\right\rangle \leq\left\langle B_{m, \sigma}, T_{m, \sigma}\right\rangle$ and

$$
\left\langle B_{m+1, \tau}, T_{m+1, \tau}\right\rangle \leq\left\langle B_{m, \sigma_{m}}, T_{m, \sigma_{m}}\right\rangle,
$$

we conclude that $\left\langle\left\langle B_{m+1, \tau}, T_{m+1, \tau}\right\rangle,\left\langle B_{m+1, \sigma}, T_{m+1, \sigma}\right\rangle\right\rangle$ is also $\langle i, j\rangle$-saturated. In the case when $\sigma=$ $\left(\sigma_{m}\right)-\langle n\rangle$ for some $n \in \omega$ and $\tau \in L\left(P_{m}\right) \backslash\left\{\sigma_{m}\right\}$, we have that $\sigma_{m}<_{\text {lex }} \tau$. Since $\sigma_{m}, \tau \in L\left(P_{m}\right)$, the induction hypothesis tells us that $\left\langle\left\langle B_{m, \tau}, T_{m, \tau}\right\rangle,\left\langle B_{m, \sigma_{m}}, T_{m, \sigma_{m}}\right\rangle\right\rangle$ is $\langle i, j\rangle$-saturated. Since we know that

$$
\left\langle B_{m+1, \tau}, T_{m+1, \tau}\right\rangle \leq\left\langle B_{m, \tau}, T_{m, \tau}\right\rangle
$$

and $\left\langle B_{m+1, \sigma}, T_{m+1, \sigma}\right\rangle \leq\left\langle B_{m, \sigma_{m}}, T_{m, \sigma_{m}}\right\rangle$, we conclude that

$$
\left\langle\left\langle B_{m+1, \tau}, T_{m+1, \tau}\right\rangle,\left\langle B_{m+1, \sigma}, T_{m+1, \sigma}\right\rangle\right\rangle
$$

is also $\langle i, j\rangle$-saturated. This verifies item 7 .

To verify item 6, fix $m^{\prime} \in \omega$ with $m^{\prime}<m+1$ and fix $\sigma \in L\left(P_{m+1}\right)$. Suppose first that $\sigma \in L\left(P_{m}\right) \backslash$ $\left\{\sigma_{m}\right\}$. If $m^{\prime}<m$, then the induction hypothesis together with the fact that $T_{m+1, \sigma} \subseteq T_{m, \sigma}$ gives what is needed. Now suppose that $m^{\prime}=m$. Then we cannot have $\sigma_{m} \subsetneq \sigma$. If $\sigma_{m}<_{\text {lex }} \sigma$, then $\sigma \in \mathcal{G}_{0}$, and by the definition of $T_{m+1, \sigma}$, for each $K \in T_{m+1, \sigma}, x_{m+1} \neq F(K)$ and $c\left(x_{m+1}, F(K)\right)=j$. Similarly, if $\sigma<_{\operatorname{lex}} \sigma_{m}$, then $\sigma \in \mathcal{G}_{1}$, and by the definition of $T_{m+1, \sigma}$, for each $K \in T_{m+1, \sigma}, x_{m+1} \neq F(K)$ and $c\left(x_{m+1}, F(K)\right)=i$. This finishes the case when $\sigma \in L\left(P_{m}\right) \backslash\left\{\sigma_{m}\right\}$. Next suppose that $\sigma=\left(\sigma_{m}\right) \frown\langle n\rangle$ for some $n \in \omega$.

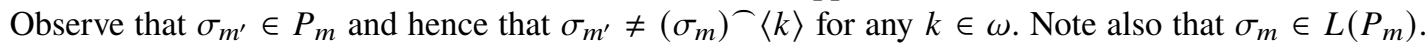
Furthermore, we know that $\left\langle B_{m+1, \sigma}, T_{m+1, \sigma}\right\rangle \leq\left\langle B_{m, \sigma_{m}}, T_{m, \sigma_{m}}\right\rangle$. Therefore, for any $K \in T_{m+1, \sigma}$, $K \cap B_{m, \sigma_{m}} \in T_{m, \sigma_{m}}$ and $F(K)=F\left(K \cap B_{m, \sigma_{m}}\right)$. Now suppose that $\sigma_{m^{\prime}} \subsetneq \sigma$. Then $\sigma_{m^{\prime}} \subseteq \sigma_{m}$. If $\sigma_{m^{\prime}}=\sigma_{m}$, then $m=m^{\prime}$, and by item 13 we have that for each $K \in T_{m+1, \sigma}, F(K) \neq x_{m+1}$ and $c\left(x_{m+1}, F(K)\right)=i$, as required. So assume that $\sigma_{m^{\prime}} \subsetneq \sigma_{m}$. Then $m^{\prime}<m$, and by the induction hypothesis, for each $K \in T_{m, \sigma_{m}}, F(K) \neq x_{m^{\prime}+1}$ and $c\left(F(K), x_{m^{\prime}+1}\right)=i$. Therefore for each $K \in T_{m+1, \sigma}$, $F(K)=F\left(K \cap B_{m, \sigma_{m}}\right) \neq x_{m^{\prime}+1}$ and $c\left(F(K), x_{m^{\prime}+1}\right)=c\left(F\left(K \cap B_{m, \sigma_{m}}\right), x_{m^{\prime}+1}\right)=i$. This finishes the case when $\sigma_{m^{\prime}} \subsetneq \sigma$. Next assume that $\sigma_{m^{\prime}}<_{\text {lex }} \sigma$. Then $\sigma_{m^{\prime}}<_{\text {lex }} \sigma_{m}$ and $m^{\prime}<m$. So by the induction 
hypothesis, for each $K \in T_{m, \sigma_{m}}, F(K) \neq x_{m^{\prime}+1}$ and $c\left(F(K), x_{m^{\prime}+1}\right)=j$. Therefore, for any $K \in T_{m+1, \sigma}$, $F(K)=F\left(K \cap B_{m, \sigma_{m}}\right) \neq x_{m^{\prime}+1}$ and $c\left(F(K), x_{m^{\prime}+1}\right)=c\left(F\left(K \cap B_{m, \sigma_{m}}\right), x_{m^{\prime}+1}\right)=j$. Finally, assume that $\sigma<_{\text {lex }} \sigma_{m^{\prime}}$. Then $\sigma_{m}<_{\operatorname{lex}} \sigma_{m^{\prime}}$ and $m^{\prime}<m$. So by the induction hypothesis, for each $K \in T_{m, \sigma_{m}}$, $F(K) \neq x_{m^{\prime}+1}$ and $c\left(F(K), x_{m^{\prime}+1}\right)=i$. Therefore, for any $K \in T_{m+1, \sigma}, F(K)=F\left(K \cap B_{m, \sigma_{m}}\right) \neq x_{m^{\prime}+1}$ and $c\left(F(K), x_{m^{\prime}+1}\right)=c\left(F\left(K \cap B_{m, \sigma_{m}}\right), x_{m^{\prime}+1}\right)=i$. This concludes the verification of item 6 .

Therefore, sequences $\left\langle x_{m+1}: m \in \omega\right\rangle$ and $\left\langle F_{m}: m \in \omega\right\rangle$ having the required properties can be constructed. This finishes the proof of the theorem.

It is not necessary for $\delta$ to be a real Woodin cardinal for our proof of Theorem 47 to work. The following is a corollary to the proof of Theorem 47 :

Corollary 48. Fix a regular topological space $\langle X, \mathcal{T}, \mathcal{B}\rangle$, where $\mathcal{B} \subseteq \mathcal{T}$ is a point-countable base and $\langle X, \mathcal{T}\rangle$ is not left-separated. Fix $l \in \omega$ with $l>0$ and fix $c:[X]^{2} \rightarrow l$. Suppose there exist $N$ and $\delta$ so that $N$ is an inner model of $\mathrm{ZFC}, \delta \in N$ is a Woodin cardinal relative to $N,\langle X, \mathcal{T}, \mathcal{B}, c\rangle \in V_{\delta}^{N}$ and $\mathcal{B}^{\omega} \subseteq N$. Then there exist $\langle i, j\rangle \in l \times l$ and a nonempty countable $Y \subseteq X$ such that $Y$ is dense in itself and $c^{\prime \prime}[Y]^{2} \subseteq\{i, j\}$.

Proof. Work in $N$. It is clear that $\langle X, \mathcal{T}\rangle$ is still a regular topological space with base $\mathcal{B} \subseteq \mathcal{T}$. For any $x \in X, \mathcal{B}_{\{x\}}=\{U \in \mathcal{B}: x \in U\}$ is a countable nonempty set in $V$, and so there is an onto function $f: \omega \rightarrow \mathcal{B}_{\{x\}}$. Note that $f \in \mathcal{B}^{\omega}$. So $f \in N$, because of the hypothesis that $\mathcal{B}^{\omega} \subseteq N$. Therefore $\mathcal{B}$ is point-countable in $N$. Next suppose that $<_{\text {wo }}$ is a well-ordering of $X$ in $N$. Then since $\langle X, \mathcal{T}\rangle$ is not left-separated in $V$ and since $<_{\text {wo }}$ well-orders $X$ in $V$, it follows that $\exists x \in X \forall U \in \mathcal{T} \exists z \in$ $X\left[\left(z \nless_{\text {wo }} x \wedge z \notin U\right) \vee\left(z \in U \wedge z<_{\text {wo }} x\right)\right]$. This is $\Delta_{0}$ and clearly relativises to $N$. Hence $\langle X, \mathcal{T}\rangle$ is not left-separated in $N$. Since $\delta$ is Woodin in $N,\langle X, \mathcal{T}, \mathcal{B}, c\rangle \in V_{\delta}^{N}$ and $c:[X]^{2} \rightarrow l$, the proof of Theorem 47 is applicable in $N$. Therefore there exist $Y \in N$ and $i, j \in l$ such that according to $N, Y$ is nonempty and countable, $Y \subseteq X, \forall y \in Y \forall U \in \mathcal{T}\left[y \in U \Longrightarrow \exists y^{\prime} \in Y\left[y^{\prime} \neq y \wedge y^{\prime} \in U\right]\right]$ and $\forall v \in[Y]^{2}[c(v)=i \vee c(v)=j]$. It is clear that all of these properties relativise up to $V$. Hence $Y$ is as required.

The hypothesis of Corollary 48 is fairly weak and does not even imply the existence of an inaccessible cardinal in $V$. Recent work in inner model theory shows that frequently occurring situations like the failure of $\square$ automatically lead to the satisfaction of Corollary 48's hypothesis for all relevant spaces and colourings. We now state some corollaries to Theorem 47. Corollary 49 establishes Theorem 17 which, as pointed out in Section 3, implies Theorem 3.

Corollary 49. Assume (*). Then for every regular topological space $\langle X, \mathcal{T}\rangle$ which is not left-separated and has a point-countable base, every $0<l<\omega$ and every colouring $c:[X]^{2} \rightarrow l$, there exists $Y \subseteq X$ such that $Y$ is homeomorphic to the rationals and c realises at most two colours on $Y$. In particular, this conclusion holds if there exists a proper class of Woodin cardinals or one strongly compact cardinal.

Proof. Let $X, \mathcal{T}, l$ and $c$ be given. Let $\mathcal{B} \subseteq \mathcal{T}$ be a point-countable base for $\langle X, \mathcal{T}\rangle$. Let $\alpha$ be so that $\langle X, \mathcal{T}, \mathcal{B}, c\rangle \in V_{\alpha}$. Use the hypothesis to find $N$ and $\delta$ such that $N$ is an inner model of ZFC, $V_{\alpha+\omega} \subseteq N$, $\delta>\alpha, \delta \in N$ and $\delta$ is a Woodin cardinal in $N$. Then $\langle X, \mathcal{T}, \mathcal{B}, c\rangle \in V_{\delta}^{N}$ and $\mathcal{B}^{\omega} \subseteq N$. Therefore the conclusion follows by applying Corollary 48.

To prove the final statement of the corollary, assume that there is a strongly compact cardinal $\kappa$. By a well-known theorem of Solovay, $\square_{\lambda^{+}}$fails for all $\lambda \geq \kappa$. By [31, Theorem .04], if $\square_{\lambda^{+}}$fails for any proper class of singular strong limit $\lambda$, then for every ordinal $\alpha$, there exists an inner model $N$ of ZFC such that $V_{\alpha} \subseteq N$ and in $N$ there exists a proper class of Woodin cardinals. The conclusion now follows exactly as in the previous paragraph.

Corollary 50. Assume the P-ideal dichotomy. Then the 2-dimensional Ramsey degree of $\mathbb{Q}$ in any regular topological space which is not left-separated and has a point-countable base is at most 2 . 
Proof. By a well-known theorem of Todorcevic [27], the P-ideal dichotomy implies the failure of $\square_{K}$ for all $\kappa \geq \omega_{1}$. Hence the conclusion can be derived by using [31, Theorem .04] like in the proof of Corollary 49.

We do not know of a direct proof of Corollary 50 that does not go through recent developments in inner model theory. Corollary 51 establishes Theorem 3 for all uncountable sets of reals. Note that a single Woodin cardinal suffices for this special case of Theorem 3, as every set of reals is a member of $V_{\delta}$ when $\delta$ is the least Woodin cardinal.

Corollary 51. Suppose that $X$ is any uncountable set of reals, that $0<l<\omega$ and that $c:[X]^{2} \rightarrow l$. Suppose there exist $N$ and $\delta$ such that $N$ is an inner model of ZFC, $X, c, \delta \in N$ and $\delta$ is a Woodin cardinal in $N$. Then there exists $Y \subseteq X$ such that $Y$ is homeomorphic to the rationals and $c$ realises at most two colours on Y. In particular, the conclusion holds if there exists at least one Woodin cardinal or one strongly compact cardinal.

Proof. Work in $N$, where $\delta$ is a Woodin cardinal. $X$ is an uncountable set of reals because it is so in $V$. Therefore if $\mathcal{T}$ is the usual topology on $X$ as calculated inside $N$, then there is in $N$ a countable base $\mathcal{B} \subseteq \mathcal{T},\langle X, \mathcal{T}\rangle$ is regular and not left-separated and $\langle X, \mathcal{T}, \mathcal{B}\rangle \in V_{\delta}^{N}$. Since $c \in N$ and $c:[X]^{2} \rightarrow l$, the proof of Theorem 47 is applicable inside $N$. So we get $Y \in N$ and $i, j \in l$ such that according to $N, Y$ is nonempty and countable, $Y \subseteq X, \forall y \in Y \forall n \in \omega \exists y^{\prime} \in Y\left[y^{\prime} \neq y \wedge\left|y^{\prime}-y\right|<\frac{1}{n+1}\right]$ and $\forall v \in[Y]^{2}[c(v)=i \vee c(v)=j]$. It is clear that all of these properties relativise up to $V$. Hence $Y$ is as required.

It is easy to modify the proof of Theorem 47 to show that the conclusion of Corollary 51 also holds if there is a precipitous ideal on $\omega_{1}$. It is not known at present whether any large-cardinal hypothesis proves the existence of a precipitous ideal on $\omega_{1}$. However, the existence of a precipitous ideal on $\omega_{1}$ is equal in consistency strength to the existence of one measurable cardinal (see [12]), which is considerably lower in consistency strength than the existence of one Woodin cardinal. Hence a measurable cardinal puts an upper bound on the consistency strength of the statement that the 2-dimensional Ramsey degree of $\mathbb{Q}$ within the class of all uncountable sets of real numbers is 2 . We do not know whether this statement has any large-cardinal strength. However, as mentioned in the introduction, it would not be surprising if the much more general Theorem 17 turns out to be equiconsistent with some large-cardinal axiom.

Acknowledgments. The first author was partially supported by the Singapore Ministry of Education's research grant number MOE2017-T2-2-125.

The second author is partially supported by grants from the Natural Sciences and Engineering Research Council (455916) and the Centre national de la recherche scientifique (IMJ-PRG UMR7586).

We thank an anonymous reader for pointing out Corollary 48 to us.

Conflict of Interest: None.

\section{References}

[1] J. E. Baumgartner, 'Partition relations for countable topological spaces', J. Combin. Theory Ser. A 43(2) (1986), 178-195.

[2] E. Borel, 'Sur la classification des ensembles de mesure nulle', Bull. Soc. Math. France 47 (1919), 97-125.

[3] D. C. Devlin, Some Partition Theorems and Ultrafilters on $\omega$, Ph.D. thesis, Dartmouth College, Ann Arbor, MI, 1980.

[4] A. Ehrenfeucht and A. Mostowski, 'Models of axiomatic theories admitting automorphisms', Fund. Math. 43 (1956), $50-68$.

[5] P. Erdôs and R. Rado, 'Combinatorial theorems on classifications of subsets of a given set', Proc. Lond. Math. Soc. (3) 2 (1952), 417-439.

[6] W. G. Fleissner, 'Left separated spaces with point-countable bases', Trans. Amer. Math. Soc. 294(2) (1986), 665-677.

[7] M. Foreman, Ideals and Generic Elementary Embeddings, Handbook of Set Theory, Vol. 2 (Springer, Dordrecht, 2010), 885-1147.

[8] M. Foreman, M. Magidor and S. Shelah, 'Martin's maximum, saturated ideals, and nonregular ultrafilters, I', Ann. of Math. (2) 127(1) (1988), 1-47.

[9] F. Galvin, Letter to R. Laver (March 19, 1970). 
[10] J. Gerlits and Z. Szentmiklóssy, 'A Ramsey-type topological theorem', Topology Appl. 125(2) (2002), 343-355.

[11] R. Haydon, Some problems about scattered spaces, Séminaire d'Initiation à l'Analyse, Publ. Math. Univ. Pierre et Marie Curie, vol. 95, Univ. Paris VI, Paris, 1989/1990, pp. Exp. No. 9, 10.

[12] T. Jech, Set Theory, third millennium edn., revised and expanded, Springer Monogr. Math. (Springer-Verlag, Berlin, 2003).

[13] R. Jensen and J. Steel, ' $K$ without the measurable', J. Symb. Log. 78(3) (2013), 708-734.

[14] A. Kanamori, The Higher Infinite, second ed., Springer Monogr. Math. (Springer-Verlag, Berlin, 2009), Large cardinals in set theory from their beginnings, MathsciNet, Paperback reprint of the 2003 edition.

[15] A. S. Kechris, V. G. Pestov and S. Todorcevic, 'Fraïssé limits, Ramsey theory, and topological dynamics of automorphism groups', Geom. Funct. Anal. 15(1) (2005), 106-189.

[16] P. B. Larson, The Stationary Tower, Univ. Lecture Ser, 32 (American Mathematical Society, Providence, RI, 2004), Notes on a course by W. Hugh Woodin.

[17] R. Laver, 'On the consistency of Borel's conjecture', Acta Math. 137(3-4) (1976), 151-169.

[18] M. Magidor, 'How large is the first strongly compact cardinal? or A study on identity crises', Ann. Math. Logic 10(1) (1976), 33-57.

[19] D. Raghavan and S. Todorcevic, 'Galvin's problem in higher dimension', In preparation.

[20] F. P. Ramsey, 'On a problem of formal logic', Proc. Lond. Math. Soc. (2) 30(4) (1929), 264-286.

[21] S. Shelah, 'Strong partition relations below the power set: Consistency; was Sierpiński right? II', in Sets, Graphs and Numbers (Budapest, 1991), Colloquia Mathematica Societatis János Bolyai, 60 (North-Holland, Amsterdam, 1992), 637-668.

[22] S. Shelah, 'Was Sierpiński right? IV', J. Symb. Log. 65(3) (2000), 1031-1054.

[23] W. Sierpiński, 'Sur une problème de la théorie des relations', Ann. Sc. Norm. Super. Pisa (2) 2 (1933), $239-242$.

[24] S. Todorcevic, 'Partition relations for partially ordered sets', Acta Math. 155(1-2) (1985), 1-25.

[25] S. Todorcevic, 'Partitioning pairs of countable ordinals', Acta Math. 159(3-4) (1987), 261-294.

[26] S. Todorcevic, 'A partition property of spaces with point-countable bases', Unpublished notes (June 1996).

[27] S. Todorcevic, 'A dichotomy for P-ideals of countable sets', Fund. Math. 166(3) (2000), 251-267.

[28] S. Todorcevic, 'Universally meager sets and principles of generic continuity and selection in Banach spaces', Adv. Math. 208(1) (2007), 274-298.

[29] S. Todorcevic, Introduction to Ramsey Spaces, Ann. of Math. Stud., 174 (Princeton University Press, Princeton, NJ, 2010).

[30] S. Todorcevic and W. Weiss, 'Partitioning metric spaces', Unpublished manuscript (September 1995).

[31] N. Trang, 'PFA and guessing models', Israel J. Math. 215(2) (2016), 607-667.

[32] W. H. Woodin, 'Supercompact cardinals, sets of reals, and weakly homogeneous trees', Proc. Natl. Acad. Sci. USA 85(18) (1988), 6587-6591.

[33] W. H. Woodin, The Axiom of Determinacy, Forcing Axioms, and the Nonstationary Ideal, de Gruyter Series in Logic and its Applications, 1 (Walter de Gruyter \& Co., Berlin, 1999). 